MATHEMATICS OF COMPUTATION

Volume 81, Number 278, April 2012, Pages 811-837

S $0025-5718(2011) 02459-2$

Article electronically published on September 28, 2011

\title{
GREEDY BISECTION GENERATES OPTIMALLY ADAPTED TRIANGULATIONS
}

\author{
JEAN-MARIE MIREBEAU AND ALBERT COHEN
}

\begin{abstract}
We study the properties of a simple greedy algorithm for the generation of data-adapted anisotropic triangulations. Given a function $f$, the algorithm produces nested triangulations $\mathcal{T}_{N}$ and corresponding piecewise polynomial approximations $f_{N}$ of $f$. The refinement procedure picks the triangle which maximizes the local $L^{p}$ approximation error, and bisects it in a direction which is chosen so to minimize this error at the next step. We study the approximation error in the $L^{p}$ norm when the algorithm is applied to $C^{2}$ functions with piecewise linear approximations. We prove that as the algorithm progresses, the triangles tend to adopt an optimal aspect ratio which is dictated by the local hessian of $f$. For convex functions, we also prove that the adaptive triangulations satisfy the convergence bound $\left\|f-f_{N}\right\|_{L^{p}} \leq C N^{-1}\left\|\sqrt{\operatorname{det}\left(d^{2} f\right)}\right\|_{L^{\tau}}$ with $\frac{1}{\tau}:=\frac{1}{p}+1$, which is known to be asymptotically optimal among all possible triangulations.
\end{abstract}

\section{INTRODUCTION}

In finite element approximation, a classical and important distinction is made between uniform and adaptive methods. In the first case all the elements which constitute the mesh have comparable shape and size, while these attributes are allowed to vary strongly in the second case. An important feature of adaptive methods is the fact that the mesh is not fixed in advance but rather tailored to the properties of the function $f$ to be approximated. Since the function approximating $f$ is not picked from a fixed linear space, adaptive finite elements can be considered as an instance of non-linear approximation. Other instances include approximation by rational functions, or by $N$-term linear combinations of a basis or dictionary. We refer to [9] for a general survey on non-linear approximation.

In this paper, we focus our interest on piecewise linear finite element functions defined over triangulations of a bidimensional polygonal domain $\Omega \subset \mathbb{R}^{2}$. Given a triangulation $\mathcal{T}$ we denote by $V_{\mathcal{T}}:=\left\{v\right.$ s.t. $\left.v_{\mid T} \in \Pi_{1}, T \in \mathcal{T}\right\}$ the associated finite element space. The norm in which we measure the approximation error is the $L^{p}$ norm for $1 \leq p \leq \infty$ and we therefore do not require that the triangulations are conforming and that the functions of $V_{\mathcal{T}}$ are continuous between triangles. For a given function $f$ we define

$$
e_{N}(f)_{L^{p}}:=\inf _{\#(\mathcal{T}) \leq N} \inf _{g \in V_{\mathcal{T}}}\|f-g\|_{L^{p}},
$$

Received by the editor October 20, 2008 and, in revised form, June 15, 2010. 2010 Mathematics Subject Classification. Primary 65-XX; Secondary 41-XX. 
the best approximation error of $f$ when using at most $N$ elements. In adaptive finite element approximation, some critical questions are:

(1) Given a function $f$ and a number $N>0$, how can we characterize the optimal mesh for $f$ with $N$ elements corresponding to the above defined best approximation error?

(2) What quantitative estimates are available for the best approximation error $e_{N}(f)_{L^{p}}$ ? Such estimates should involve the derivatives of $f$ in a different way than for non-adaptive meshes.

(3) Can we build, by a simple algorithmic procedure, a mesh $\mathcal{T}_{N}$ of cardinality $N$ and a finite element function $f_{N} \in V_{\mathcal{T}_{N}}$ such that $\left\|f-f_{N}\right\|_{L^{p}}$ is comparable to $e_{N}(f)_{L^{p}}$ ?

While the optimal mesh is usually difficult to characterize exactly, it should satisfy two intuitively desirable features: (i) the triangulation should equidistribute the local approximation error between each triangle and (ii) the aspect ratio of a triangle $T$ should be isotropic with respect to a distorted metric induced by the local value of the hessian $d^{2} f$ on $T$ (and therefore anisotropic in the sense of the euclidean metric). Under such prescriptions on the mesh, quantitative error estimates have recently been obtained in [7, 1, when $f$ is a $C^{2}$ function. These estimates are of the form

$$
e_{N}(f)_{L^{p}} \leq C N^{-1}\left\|\sqrt{\left|\operatorname{det}\left(d^{2} f\right)\right|}\right\|_{L^{\tau}}, \frac{1}{\tau}=\frac{1}{p}+1,
$$

where $\operatorname{det}\left(d^{2} f\right)$ is the determinant of the $2 \times 2$ hessian matrix. For a convex $C^{2}$ function $f$ this estimate has been proved to be asymptotically optimal in [7, in the following sense:

$$
\liminf _{N \rightarrow+\infty} N e_{N}(f)_{L^{p}} \geq c\left\|\sqrt{\left|\operatorname{det}\left(d^{2} f\right)\right|}\right\|_{L^{\tau}} .
$$

The convexity assumption can actually be replaced by a mild assumption on the sequence of triangulations which is used for the approximation of $f$ : a sequence $\left(\mathcal{T}_{N}\right)_{N \geq N_{0}}$ is said to be admissible if $\#\left(\mathcal{T}_{N}\right) \leq N$ and

$$
\sup _{N \geq N_{0}}\left\{N^{1 / 2} \max _{T \in \mathcal{T}_{N}} \operatorname{diam}(T)\right\}<\infty .
$$

Then it is proved in [10], that for any admissible sequence and any $C^{2}$ function $f$, one has

$$
\liminf _{N \rightarrow+\infty} N \inf _{g \in V_{\mathcal{T}_{N}}}\|f-g\|_{L^{p}} \geq c\left\|\sqrt{\left|\operatorname{det}\left(d^{2} f\right)\right|}\right\|_{L^{\tau}}
$$

The admissibility assumption is not a severe limitation for an upper estimate of the error since it is also proved that for all $\varepsilon>0$, there exist an admissible sequence such that

$$
\limsup _{N \rightarrow+\infty} N \inf _{g \in V_{\mathcal{T}_{N}}}\|f-g\|_{L^{p}} \leq C\left\|\sqrt{\left|\operatorname{det}\left(d^{2} f\right)\right|}\right\|_{L^{\tau}}+\varepsilon .
$$

We also refer to [10] for a generalization of such upper and lower estimates for higher order elements.

From the computational viewpoint, a commonly used strategy for designing an optimal mesh consists therefore in evaluating the hessian $d^{2} f$ and imposing that each triangle of the mesh is isotropic with respect to a metric which is properly related to its local value. We refer, in particular, to [3] where this program is executed 
using Delaunay mesh generation techniques. While these algorithms quickly produce anisotropic meshes which are naturally adapted to the approximated function, they suffer from two intrinsic limitations:

(1) They use the data of $d^{2} f$, and therefore do not apply to non-smooth or noisy functions.

(2) They are non-hierarchical: for $N>M$, the triangulation $\mathcal{T}_{N}$ is not a refinement of $\mathcal{T}_{M}$.

In $[8$, an alternate strategy was proposed for the design of adaptive hierarchical meshes, based on a simple greedy algorithm: starting from an initial triangulation $\mathcal{T}_{N_{0}}$, the algorithm picks the triangle $T \in \mathcal{T}_{k}$ with the largest local $L^{p}$ error. This triangle is then bisected from the mid-point of one of its edges to the opposite vertex. The choice of the edge among the three options is the one that minimizes the new approximation error after bisection. The algorithm can be applied to any $L^{p}$ function, smooth or not, in the context of piecewise polynomial approximation of any given order. In the case of piecewise linear approximation, numerical experiments in 8 indicate that this elementary strategy generates triangles with an optimal aspect ratio and approximations $f_{N} \in V_{\mathcal{T}_{N}}$ such that $\left\|f-f_{N}\right\|_{L^{p}}$ satisfies the same estimate as $e_{N}(f)_{L^{p}}$ in (1.1).

The goal of this paper is to support these experimental observations by a rigorous analysis. Our paper is organized as follows:

In $\S 2$, we introduce notations which are used throughout the paper and collect some available approximation theory results for piecewise linear finite elements, making the distinction between (i) uniform, (ii) adaptive isotropic and (iii) adaptive anisotropic triangulations. In the last case, which is in the scope of this paper, we introduce a measure of non-degeneracy of a triangle $T$ with respect to a quadratic form. We show that the optimal error estimate (1.1) is met when each triangle is non-degenerate in the sense of the above measure with respect to the quadratic form given by the local hessian $d^{2} f$. We end by briefly recalling the greedy algorithm which was introduced in 8 .

In $\S 3$, we study the behavior of the refinement procedure when applied to a quadratic function $q$ such that its associated quadratic form $\mathbf{q}$ is of positive or negative sign. A key observation is that the edge which is bisected is the longest with respect to the metric induced by $\mathbf{q}$. This allows us to prove that the triangles generated by the refinement procedure adopt an optimal aspect ratio in the sense of the non-degeneracy measure introduced in $\S 2$.

In $\S 4$, we study the behavior of the algorithm when applied to a general $C^{2}$ function $f$ which is assumed to be strictly convex (or strictly concave). We first establish a perturbation result, which shows that when $f$ is locally close to a quadratic function $q$ the algorithm behaves in a similar manner as when applied to $q$. We then prove that the diameters of the triangles produced by the algorithm tend to zero so that the perturbation result can be applied. This allows us to show that the optimal convergence estimate,

$$
\left\|f-f_{N}\right\|_{L^{p}} \leq C N^{-1}\left\|\sqrt{\left|\operatorname{det}\left(d^{2} f\right)\right|}\right\|_{L^{\tau}},
$$

is met by the sequence of approximations $f_{N} \in V_{\mathcal{T}_{N}}$ generated by the algorithm.

The extension of this result to an arbitrary $C^{2}$ function $f$ remains an open problem. It is possible to proceed to an analysis similar to $\S 3$ in the case where the quadratic form $\mathbf{q}$ is of mixed sign, also proving that the triangles adopt an 
optimal aspect ratio as they get refined. We describe this analysis in an appendix. However, it seems difficult to extend the perturbation analysis of $\S 4$ to this new setting. In particular the diameters of the triangles are no more ensured to tend to zero, and one can even exhibit examples of non-convex $C^{2}$ functions $f$ for which the approximation $f_{N}$ fails to converge towards $f$ due to this phenomenon. Such examples are discussed in [8] which also proposes a modification of the algorithm for which convergence is always ensured. However, we do not know if the optimal convergence estimate (1.5) holds for any $f \in \mathcal{C}^{2}$ with this modified algorithm, although this seems plausible from the numerical experiments.

\section{Adaptive finite Element approximation}

2.1. Notation. We shall make use of a linear approximation operator $\mathcal{A}_{T}$ that maps continuous functions defined on $T$ onto $\Pi_{1}$. For an arbitrary but fixed $1 \leq$ $p \leq \infty$, we define the local $L^{p}$ approximation error

$$
e_{T}(f)_{p}:=\left\|f-\mathcal{A}_{T} f\right\|_{L^{p}(T)} .
$$

The critical assumptions in our analysis for the operator $\mathcal{A}_{T}$ will be the following:

(1) $\mathcal{A}_{T}$ is continuous in the $L^{\infty}$ norm.

(2) $\mathcal{A}_{T}$ commutes with affine changes of variables: $\mathcal{A}_{T}(f) \circ \phi=\mathcal{A}_{\phi^{-1}(T)}(f \circ \phi)$ for all affine $\phi$.

(3) $\mathcal{A}_{T}$ reproduces $\Pi_{1}: \mathcal{A}_{T}(\pi)=\pi$, for any $\pi \in \Pi_{1}$.

Note that the commutation assumption implies that for any function $f$ and any affine transformation $\phi: x \mapsto x_{0}+L x$ we have

$$
e_{\phi(T)}(f)_{p}=|\operatorname{det}(L)|^{1 / p} e_{T}(f \circ \phi)_{p},
$$

Two particularly simple admissible choices of approximation operators are the following:

- $\mathcal{A}_{T}=P_{T}$, the $L^{2}(T)$-orthogonal projection operator: $\int_{T}\left(f-P_{T} f\right) \pi=0$ for all $\pi \in \Pi_{1}$.

- $\mathcal{A}_{T}=I_{T}$, the local interpolation operator: $I_{T} f\left(v_{i}\right)=f\left(v_{i}\right)$ with $\left\{v_{0}, v_{1}, v_{2}\right\}$ the vertices of $T$.

All our results are simultaneously valid when $\mathcal{A}_{T}$ is either $P_{T}$ or $I_{T}$, or any linear operator that fulfills the three above assumptions.

Given a function $f$ and a triangulation $\mathcal{T}_{N}$ with $N=\#\left(\mathcal{T}_{N}\right)$, we can associate a finite element approximation $f_{N}$ defined on each $T \in \mathcal{T}_{N}$ by $f_{N}(x)=\mathcal{A}_{T} f(x)$. The global approximation error is given by

$$
\left\|f-f_{N}\right\|_{L^{p}}=\left(\sum_{T \in \mathcal{T}_{N}} e_{T}(f)_{p}^{p}\right)^{\frac{1}{p}},
$$

with the usual modification when $p=\infty$.

Remark 2.1. The operator $\mathcal{B}_{T}$ of best $L^{p}(T)$ approximation which is defined by

$$
\left\|f-\mathcal{B}_{T} f\right\|_{L^{p}(T)}=\min _{\pi \in \Pi_{m}}\|f-\pi\|_{L^{p}(T)},
$$

does not fall in the above category of operators, since it is non-linear (and not easy to compute) when $p \neq 2$. However, it is clear that any estimate on $\left\|f-f_{N}\right\|_{L^{p}}$ with $f_{N}$ defined as $\mathcal{A}_{T} f$ on each $T$ implies a similar estimate when $f_{N}$ is defined as $\mathcal{B}_{T} f$ on each $T$. 
Here and throughout the paper, when

$$
q(x, y)=a_{2,0} x^{2}+2 a_{1,1} x y+a_{0,2} y^{2}+a_{1,0} x+a_{0,1} y+a_{0,0}
$$

we denote by $\mathbf{q}$ the associated quadratic form: if $u=(x, y)$, then

$$
\mathbf{q}(u)=a_{2,0} x^{2}+2 a_{1,1} x y+a_{0,2} y^{2} .
$$

Note that $\mathbf{q}(u)=\langle Q u, u\rangle$ where $Q=\left(\begin{array}{ll}a_{2,0} & a_{1,1} \\ a_{1,1} & a_{0,2}\end{array}\right)$. We define

$$
\operatorname{det}(\mathbf{q}):=\operatorname{det}(Q) \text {. }
$$

If $\mathbf{q}$ is a positive or negative quadratic form, we define the $\mathbf{q}$-metric

$$
|v|_{\mathbf{q}}:=\sqrt{|\mathbf{q}(v)|}
$$

which coincides with the euclidean norm when $\mathbf{q}(v)=x^{2}+y^{2}$ for $v=(x, y)$. If $\mathbf{q}$ is a quadratic form of mixed sign, we define the associated positive form $|\mathbf{q}|$ which corresponds to the symmetric matrix $|Q|$ that has the same eigenvectors as $Q$ with eigenvalues $(|\lambda|,|\mu|)$ if $(\lambda, \mu)$ are the eigenvalues of $Q$. Note that generally $|\mathbf{q}|(u) \neq|\mathbf{q}(u)|$ and that one always has $|\mathbf{q}(u)| \leq|\mathbf{q}|(u)$.

Remark 2.2. If $\operatorname{det} Q>0$, then there exists a $2 \times 2$ matrix $L$ and $\varepsilon \in\{+1,-1\}$ such that

$$
L^{t} Q L=\varepsilon\left(\begin{array}{ll}
1 & 0 \\
0 & 1
\end{array}\right) .
$$

The linear change of coordinates $\phi(u):=L u$, where $u=(x, y) \in \mathbb{R}^{2}$, therefore satisfies $\mathbf{q} \circ \phi(u)=\varepsilon\left(x^{2}+y^{2}\right)$. On the other hand, if $\operatorname{det} Q<0$, then there exists a $2 \times 2$ matrix $L$ such that

$$
L^{t} Q L=\left(\begin{array}{cc}
1 & 0 \\
0 & -1
\end{array}\right)
$$

Defining again $\phi(u):=L u$ we obtain in this case $\mathbf{q} \circ \phi(u)=x^{2}-y^{2}$.

2.2. From uniform to adaptive isotropic triangulations. A standard estimate in finite element approximation states that if $f \in W^{2, p}(\Omega)$, then

$$
\inf _{g \in V_{h}}\|f-g\|_{L^{p}} \leq C h^{2}\left\|d^{2} f\right\|_{L^{p}}
$$

where $V_{h}$ is the piecewise linear finite element space associated with a triangulation $\mathcal{T}_{h}$ of mesh size $h:=\max _{T \in \mathcal{T}_{h}} \operatorname{diam}(T)$. If we restrict our attention to uniform triangulations, we have

$$
N:=\#\left(\mathcal{T}_{h}\right) \sim h^{-2} .
$$

Therefore, denoting by $e_{N}^{\text {unif }}(f)_{L^{p}}$ the $L^{p}$ approximation error by a uniform triangulation of cardinality $N$, we can re-express the above estimate as

$$
e_{N}^{\text {unif }}(f)_{L^{p}} \leq C N^{-1}\left\|d^{2} f\right\|_{L^{p}}
$$

This estimate can be significantly improved when using adaptive partitions. We give here some heuristic arguments, which are based on the assumption that on each triangle $T$ the relative variation of $d^{2} f$ is small so that it can be considered as a constant over $T$ (which means that $f$ is replaced by a quadratic function on each $T$ ), and we also indicate the available results which are proved more rigorously.

First consider isotropic triangulations, i.e., such that all triangles satisfy a uniform estimate

$$
\rho_{T}=\frac{h_{T}}{r_{T}} \leq A,
$$


where $h_{T}:=\operatorname{diam}(T)$ the size of its longest edge, and $r_{T}$ is the radius of the largest disc contained in $T$. In such a case we start from the local approximation estimate on any $T$,

$$
e_{T}(f)_{p} \leq C h_{T}^{2}\left\|d^{2} f\right\|_{L^{p}(T)},
$$

and notice that

$$
h_{T}^{2}\left\|d^{2} f\right\|_{L^{p}(T)} \sim|T|\left\|d^{2} f\right\|_{L^{p}(T)}=\left\|d^{2} f\right\|_{L^{\tau}(T)},
$$

with $\frac{1}{\tau}:=\frac{1}{p}+1$ and $|T|$ the area of $T$, where we have used the isotropy assumption (2.4) in the equivalence and the fact that $d^{2} f$ is constant over $T$ in the equality. It follows that

$$
e_{T}(f)_{p} \leq C\left\|d^{2} f\right\|_{L^{\tau}(T)}, \quad \frac{1}{\tau}:=\frac{1}{p}+1 .
$$

Assume now that we can construct adaptive isotropic triangulations $\mathcal{T}_{N}$ with $N:=$ $\#\left(\mathcal{T}_{N}\right)$ which equidistributes the local error in the sense that for some prescribed $\varepsilon>0$

$$
c \varepsilon \leq e_{T}(f)_{p} \leq \varepsilon,
$$

with $c>0$ a fixed constant independent of $T$ and $N$. Then defining $f_{N}$ as $\mathcal{A}_{T}(f)$ on each $T \in \mathcal{T}_{N}$, we have on the one hand,

$$
\left\|f-f_{N}\right\|_{L^{p}} \leq N^{1 / p} \varepsilon
$$

and on the other hand, with $\frac{1}{\tau}:=\frac{1}{p}+1$,

$$
N(c \varepsilon)^{\tau} \leq \sum_{T \in \mathcal{T}_{N}}\left\|f-f_{N}\right\|_{L^{p}(T)}^{\tau} \leq C^{\tau} \sum_{T \in \mathcal{T}_{N}}\left\|d^{2} f\right\|_{L^{\tau}(T)}^{\tau} \leq C^{\tau}\left\|d^{2} f\right\|_{L^{\tau}}^{\tau}
$$

Combining both, one obtains for $e_{N}^{\text {iso }}(f)_{L^{p}}:=\left\|f-f_{N}\right\|_{L^{p}}$ the estimate

$$
e_{N}^{\text {iso }}(f)_{L^{p}} \leq C N^{-1}\left\|d^{2} f\right\|_{L^{\tau}}
$$

This estimate improves upon (2.3) since the rate $N^{-1}$ is now obtained with the weaker smoothness condition $d^{2} f \in L^{\tau}$ and since, even for smooth $f$, the quantity $\left\|d^{2} f\right\|_{L^{\tau}}$ might be significantly smaller than $\left\|d^{2} f\right\|_{L^{p}}$. This type of result is classical in non-linear approximation and also occurs when we consider best $N$-term approximation in a wavelet basis.

The principle of error equidistribution suggests a simple greedy algorithm to build an adaptive isotropic triangulation for a given $f$, similar to our algorithm but where the bisection of the triangle $T$ that maximizes the local error $e_{T}(f)_{p}$ is systematically done from its most recently created vertex in order to preserve the estimate (2.4). Such an algorithm cannot exactly equilibrate the error in the sense of (2.5) and therefore does not lead to the same the optimal estimate as in (2.6). However, it was proved in [2] that it satisfies

$$
\left\|f-f_{N}\right\|_{L^{p}} \leq C|f|_{B_{\tau, \tau}^{2}} N^{-1},
$$

for all $\tau$ such that $\frac{1}{\tau}<\frac{1}{p}+1$, provided that the local approximation operator $\mathcal{A}_{T}$ is bounded in the $L^{p}$ norm. Here $B_{\tau, \tau}^{2}$ denotes the usual Besov space which is a natural substitute for $W^{2, \tau}$ when $\tau<1$. Therefore this estimate is not far from (2.6). 
2.3. Anisotropic triangulations: the optimal aspect ratio. We now turn to anisotropic adaptive triangulations, and start by discussing the optimal shape of a triangle $T$ for a given function $f$ at a given point. For this purpose, we again replace $f$ by a quadratic function assuming that $d^{2} f$ is constant over $T$. For such a $q \in \Pi_{2}$ and its associated quadratic form $\mathbf{q}$, we first derive an equivalent quantity for the local approximation error. Here and as well as in $\S 3$ and $\S 4$, we consider a triangle $T$ and we denote by $(a, b, c)$ its edge vectors oriented in clockwise or anti-clockwise direction so that

$$
a+b+c=0 .
$$

Proposition 2.3. The local $L^{p}$-approximation error satisfies

$$
e_{T}(q)_{p}=e_{T}(\mathbf{q})_{p} \sim|T|^{\frac{1}{p}} \max \{|\mathbf{q}(a)|,|\mathbf{q}(b)|,|\mathbf{q}(c)|\},
$$

where the constant in the equivalence is independent of $q, T$ and $p$.

Proof. The first equality is trivial since $q$ and $\mathbf{q}$ differ by an affine function. Let $T_{\text {eq }}$ be an equilateral triangle of area $\left|T_{\text {eq }}\right|=1$, and edges $a, b, c$. Let $E$ be the 3 -dimensional vector space of all quadratic forms. Then the following quantities are norms on $E$, and thus equivalent:

$$
e_{T_{\mathrm{eq}}}(\mathbf{q})_{p} \sim \max \{|\mathbf{q}(a)|,|\mathbf{q}(b)|,|\mathbf{q}(c)|\} .
$$

Note that the constants in this equivalence are independent of $p$ since all $L^{p}(T)$ norms are uniformly equivalent on $E$.

If $T$ is an arbitrary triangle, there exists an affine transform $\phi: x \mapsto x_{0}+L x$ such that $T=\phi\left(T_{\mathrm{eq}}\right)$. For any quadratic function $q$, we thus obtain from (2.1)

$$
e_{T}(\mathbf{q})=e_{T}(q)=e_{\phi\left(T_{\mathrm{eq}}\right)}(q)=|\operatorname{det} L|^{\frac{1}{p}} e_{T_{\mathrm{eq}}}(q \circ \phi)=|\operatorname{det} L|^{\frac{1}{p}} e_{T_{\mathrm{eq}}}(\mathbf{q} \circ L)
$$

since $\mathbf{q} \circ L$ is the homogeneous part of $q \circ \phi$. By (2.7), we thus have

$$
e_{T}(\mathbf{q}) \sim|\operatorname{det} L|^{\frac{1}{p}} \max \{|\mathbf{q}(L a)|,|\mathbf{q}(L b)|,|\mathbf{q}(L c)|\},
$$

where $\{a, b, c\}$ are again the edge vectors of $T_{\text {eq }}$. Remarking that $|T|=|\operatorname{det} L|$ and that $\{L a, L b, L c\}$ are the edge vectors of $T$, this concludes the proof of this proposition.

In order to describe the optimal shape of a triangle $T$ for the quadratic function $q$, we fix the area of $|T|$ and try to minimize the error $e_{T}(q)_{p}$ or, equivalently, $\max \{|\mathbf{q}(a)|,|\mathbf{q}(b)|,|\mathbf{q}(c)|\}$. The solution to this problem can be found by introducing for any $\mathbf{q}$ such that $\operatorname{det}(\mathbf{q}) \neq 0$ the following measure of non-degeneracy for $T$ :

$$
\rho_{\mathbf{q}}(T):=\frac{\max \{|\mathbf{q}(a)|,|\mathbf{q}(b)|,|\mathbf{q}(c)|\}}{|T| \sqrt{|\operatorname{det}(\mathbf{q})|}}
$$

Let $\phi$ be a linear change of variables, $\mathbf{q}$ a quadratic form and $T$ a triangle of edges $a, b, c$. Then $\operatorname{det}(\mathbf{q} \circ \phi)=(\operatorname{det} \phi)^{2} \operatorname{det}(\mathbf{q})$, the edges of $\phi(T)$ are $\phi(a), \phi(b), \phi(c)$ and $|\phi(T)|=|\operatorname{det} \phi||T|$. Hence we obtain

$$
\begin{aligned}
\rho_{\mathbf{q} \circ \phi}(T) & =\frac{\max \{|\mathbf{q} \circ \phi(a)|,|\mathbf{q} \circ \phi(b)|,|\mathbf{q} \circ \phi(c)|\}}{|T| \sqrt{|\operatorname{det}(\mathbf{q} \circ \phi)|}} \\
& =\frac{\max \{|\mathbf{q}(\phi(a))|,|\mathbf{q}(\phi(b))|,|\mathbf{q}(\phi(c))|\}}{|\operatorname{det} \phi||T| \sqrt{|\operatorname{det}(\mathbf{q})|}}=\rho_{\mathbf{q}}(\phi(T)) .
\end{aligned}
$$

The last equation, combined with Remark 2.2. allows us to reduce the study of $\rho_{\mathbf{q}}(T)$ to two elementary cases by a change of variable: 
(1) The case where $\operatorname{det}(\mathbf{q})>0$ is reduced to $\mathbf{q}(x, y)=x^{2}+y^{2}$. Recall that for any triangle $T$ with edges $a, b, c$ we define $h_{T}:=\operatorname{diam}(T)=$ $\max \{|a|,|b|,|c|\}$, with $|\cdot|$ the euclidean norm. In this case we therefore have $\rho_{\mathbf{q}}(T)=\frac{h_{T}^{2}}{|T|}$, which corresponds to a standard measure of shape regularity in the sense that its boundedness is equivalent to a property such as (2.4). This quantity is minimized when the triangle $T$ is equilateral, with minimal value $\frac{4}{\sqrt{3}}$ (in fact, it was also proved in [5] that the minimum of the interpolation error $\left\|\mathbf{q}-I_{T} \mathbf{q}\right\|_{L^{p}(T)}$ among all triangles of area $|T|=1$ is attained when $T$ is equilateral). For a general quadratic form $\mathbf{q}$ of positive sign, we obtain by change of variable that the minimal value $\frac{4}{\sqrt{3}}$ is obtained for triangles which are equilateral with respect to the metric $|\cdot|_{\mathbf{q}}$. More generally, triangles with a good aspect ratio, i.e., a small value of $\rho_{\mathbf{q}}(T)$, are those which are isotropic with respect to this metric. Of course, a similar conclusion holds for a quadratic form of negative sign.

(2) The case where $\operatorname{det}(\mathbf{q})<0$ is reduced to $\mathbf{q}(x, y)=x^{2}-y^{2}$. In this case, the analysis presented in [4] shows that the quantity $\rho_{\mathbf{q}}(T)$ is minimized when $T$ is a half of a square with sides parallel to the $x$ and $y$ axes, with minimal value 2. But using (2.9) we also notice that $\rho_{\mathbf{q}}(T)=\rho_{\mathbf{q}}(L(T))$ for any linear transformation $L$ such that $\mathbf{q}=\mathbf{q} \circ L$. This holds if $L$ has eigenvalues $\left(\lambda, \frac{1}{\lambda}\right)$, where $\lambda \neq 0$, and eigenvectors $(1,1)$ and $(-1,1)$. Therefore, all images of the half square by such transformations $L$ are also optimal triangles. Note that such triangles can be highly anisotropic. For a general quadratic form $\mathbf{q}$ of mixed sign, we notice that $\rho_{\mathbf{q}}(T) \leq \rho_{|\mathbf{q}|}(T)$, and therefore triangles which are equilateral with respect to the metric $|\cdot|_{|\mathbf{q}|}$ have a good aspect ratio, i.e., a small value of $\rho_{\mathbf{q}}(T)$. In addition, by similar arguments, we find that all images of such triangles by linear transforms $L$ with eigenvalues $\left(\lambda, \frac{1}{\lambda}\right)$ and eigenvectors $(u, v)$ such that $\mathbf{q}(u)=\mathbf{q}(v)=0$ also have a good aspect ratio, since $\mathbf{q}=\mathbf{q} \circ L$ for such transforms.

We leave aside the special case where $\operatorname{det}(\mathbf{q})=0$. In such a case, the triangles minimizing the error for a given area degenerate in the sense that they should be infinitely long and thin, aligned with the direction of the null eigenvalue of $\mathbf{q}$.

Summing up, we find that triangles with a good aspect ratio are characterized by the fact that $\rho_{\mathbf{q}}(T)$ is small. In addition, from Proposition 2.3 and the definition of $\rho_{\mathbf{q}}(T)$, we have

$$
e_{T}(q)_{p} \sim|T|^{1+\frac{1}{p}} \sqrt{|\operatorname{det}(\mathbf{q})|} \rho_{\mathbf{q}}(T)=\|\sqrt{|\operatorname{det}(\mathbf{q})|}\|_{L^{\tau}(T)} \rho_{\mathbf{q}}(T), \quad \frac{1}{\tau}:=\frac{1}{p}+1 .
$$

We now return to a function $f$ such that $d^{2} f$ is assumed to be constant on every $T \in \mathcal{T}_{N}$. Assuming that all triangles have a good aspect ratio in the sense that

$$
\rho_{\mathbf{q}}(T) \leq C
$$

for some fixed constant $C$ and with $\mathbf{q}$ the value of $d^{2} f$ over $T$, we find up to a change in $C$ that

$$
e_{T}(f)_{p} \leq C\left\|\sqrt{\left|\operatorname{det}\left(d^{2} f\right)\right|}\right\|_{L^{\tau}(T)} .
$$

By similar reasoning as for isotropic triangulations, we now obtain that if the triangulation equidistributes the error in the sense of (2.5), then

$$
\left\|f-f_{N}\right\|_{L^{p}} \leq C N^{-1}\left\|\sqrt{\left|\operatorname{det}\left(d^{2} f\right)\right|}\right\|_{L^{\tau}},
$$


and therefore (1.1) holds. This estimate improves upon (2.6) since the quantity $\left\|\sqrt{\left|\operatorname{det}\left(d^{2} f\right)\right|}\right\|_{L^{\tau}}$ might be significantly smaller than $\left\|d^{2} f\right\|_{L^{\tau}}$, in particular, when $f$ has some anisotropic features, such as sharp gradients along curved edges.

The above derivation of (1.1) is heuristic and non-rigorous. Clearly, this estimate cannot be valid as such since $\operatorname{det}\left(d^{2} f\right)$ may vanish while the approximation error does not (consider for instance $f$ depending only on a single variable). More rigorous versions were derived in [7] and [1. In these results $\left|d^{2} f\right|$ is typically replaced by a majorant $\left|d^{2} f\right|+\varepsilon I$, avoiding that its determinant vanishes. The estimate (1.1) can then be rigorously proved but holds for $N \geq N(\varepsilon, f)$ large enough. This limitation is unavoidable and reflects the fact that enough resolution is needed so that the hessian can be viewed as locally constant over each optimized triangle. Another formulation, which is rigorously proved in [10], reads as follows.

Proposition 2.4. There exists an absolute constant $C>0$ such that for any polygonal domain $\Omega$ and any function $f \in C^{2}(\Omega)$, one has

$$
\limsup _{N \rightarrow+\infty} N e_{N}(f)_{L^{p}} \leq C\left\|\sqrt{\left|\operatorname{det}\left(d^{2} f\right)\right|}\right\|_{L^{\tau}} .
$$

2.4. The greedy algorithm. Given a target function $f$, our algorithm iteratively builds triangulations $\mathcal{T}_{N}$ with $N=\#\left(\mathcal{T}_{N}\right)$ and finite element approximations $f_{N}$. The starting point is a coarse triangulation $\mathcal{T}_{N_{0}}$. Given $\mathcal{T}_{N}$, the algorithm selects the triangle $T$ which maximizes the local error $e_{T}(f)_{p}$ among all triangles of $\mathcal{T}_{N}$, and bisects it from the mid-point of one of its edges towards the opposite vertex. This gives the new triangulation $\mathcal{T}_{N+1}$.

The critical part of the algorithm lies in the choice of the edge $e \in\{a, b, c\}$ from which $T$ is bisected. Denoting by $T_{e}^{1}$ and $T_{e}^{2}$ the two resulting triangles, we choose $e$ as the minimizer of a decision function $d_{T}(e, f)$, which role is to drive the generated triangles towards an optimal aspect ratio. While the most natural choice for $d_{T}(e, f)$ corresponds to the split that minimizes the error after bisection, namely

$$
d_{T}(e, f)=e_{T_{e}^{1}}(f)_{p}^{p}+e_{T_{e}^{2}}(f)_{p}^{p},
$$

we shall instead focus our attention on a decision function which is defined as the $L^{1}$ norm of the interpolation error

$$
d_{T}(e, f)=\left\|f-I_{T_{e}^{1}} f\right\|_{L^{1}\left(T_{e}^{1}\right)}+\left\|f-I_{T_{e}^{2}} f\right\|_{L^{1}\left(T_{e}^{2}\right)} .
$$

For this decision, the analysis of the algorithm is made simpler, due to the fact that we can derive explicit expressions of $\left\|f-I_{T} f\right\|_{L^{1}(T)}$ when $f=q$ is a quadratic polynomial with a positive homogeneous part $\mathbf{q}$. We prove in $\S 3$ that this choice leads to triangles with an optimal aspect ratio in the sense of a small $\rho_{\mathbf{q}}(T)$. This leads us in $\S 4$ to a proof that the algorithm satisfies the optimal convergence estimate (2.12) in the case where $f$ is $C^{2}$ and strictly convex.

Remark 2.5. It should be well understood that while the decision function is based on the $L^{1}$ norm, the selection of the triangle to be bisected is done by maximizing $e_{T}(f)_{p}$. The algorithm remains therefore governed by the $L^{p}$ norm in which we wish to minimize the error $\left\|f-f_{N}\right\|_{p}$ for a given number of triangles. Intuitively, this means that the $L^{p}$ norm influences the size of the triangles which have to equidistribute the error, but not their optimal shape. 
Remark 2.6. It was pointed out to us that the $L^{1}$ norm of the interpolation error to a suitable convex function is also used to improve the mesh in the context of moving grid techniques; see [6].

We define a variant of the decision function as follows:

$$
D_{T}(e, f):=\left\|f-I_{T} f\right\|_{L^{1}(T)}-d_{T}(e, f) .
$$

Note that $D_{T}(e, f)$ is the reduction of the $L^{1}$ interpolation error resulting from the bisection of the edge $e$, and that the selected edge that minimizes $d_{T}(\cdot, f)$ is also the one that maximizes $D(\cdot, f)$. The function $D_{T}$ has a simple expression in the case where $f$ is a convex function.

Lemma 2.7. Let $T$ be a triangle and let $f$ be a convex function on $T$. Let $e$ be an edge of $T$ with endpoints $z_{0}$ and $z_{1}$. Then

$$
D_{T}(e, f)=\frac{|T|}{3}\left(\frac{f\left(z_{0}\right)+f\left(z_{1}\right)}{2}-f\left(\frac{z_{0}+z_{1}}{2}\right)\right) .
$$

If, in addition, $f$ has $C^{2}$ smoothness, we also have

$$
D_{T}(e, f)=\frac{|T|}{6} \int_{0}^{1}\left\langle d^{2} f\left(z_{t}\right) e, e\right\rangle \min \{t, 1-t\} d t, \text { where } z_{t}:=(1-t) z_{0}+t z_{1} .
$$

Proof. Since $f$ is convex, we have $I_{T} f \geq f$ on $T$, hence

$$
\left\|f-I_{T} f\right\|_{L^{1}(T)}=\int_{T}\left(I_{T} f-f\right) .
$$

Similarly, $I_{T_{e}^{1}} f \geq f$ on $T_{e}^{1}$ and $I_{T_{e}^{2}} f \geq f$ on $T_{e}^{2}$, hence

$$
D_{T}(e, f)=\int_{T} I_{T} f-\int_{T_{e}^{1}} I_{T_{e}^{1}} f-\int_{T_{e}^{2}} I_{T_{e}^{2}} f .
$$

Let $z_{2}$ be the vertex of $T$ opposite the edge $e$. Since the function $f$ is convex, it follows the previous expression that $D_{T}(e, f)$ is the volume of the tetrahedron of vertices

$$
\left(\frac{z_{0, x}+z_{1, x}}{2}, \frac{z_{0, x}+z_{1, x}}{2}, f\left(\frac{z_{0}+z_{1}}{2}\right)\right) \text { and }\left(z_{i, x}, z_{i, y}, f\left(z_{i}\right)\right) \text { for } i=0,1,2 .
$$

where $\left(z_{i, x}, z_{i, y}\right)$ are the coordinates of $z_{i}$. Let $u=z_{0}-z_{2}$ and $v=z_{1}-z_{2}$. We thus have $D_{T}(e, f)=\frac{1}{6}|\operatorname{det}(M)|$ where

$$
M:=\left(\begin{array}{ccc}
u_{x} & v_{x} & \frac{u_{x}+v_{x}}{2} \\
u_{y} & v_{y} & \frac{u_{y}+v_{y}}{2} \\
f\left(z_{0}\right)-f\left(z_{2}\right) & f\left(z_{1}\right)-f\left(z_{2}\right) & f\left(\frac{z_{0}+z_{1}}{2}\right)-f\left(z_{2}\right)
\end{array}\right) .
$$

Subtracting the half of the first two columns to the third one we find that $M$ has the same determinant as

$$
\tilde{M}:=\left(\begin{array}{ccc}
u_{x} & v_{x} & 0 \\
u_{y} & v_{y} & 0 \\
f\left(z_{0}\right)-f\left(z_{2}\right) & f\left(z_{1}\right)-f\left(z_{2}\right) & f\left(\frac{z_{0}+z_{1}}{2}\right)-\frac{f\left(z_{0}\right)+f\left(z_{1}\right)}{2}
\end{array}\right) .
$$

Recalling that $2|T|=|\operatorname{det}(u, v)|$ we therefore obtain (2.14). In order to establish (2.15), we observe that we have in the distribution sense $\partial_{t}^{2}\left(\min \{t, 1-t\}^{+}\right)=$ 
$\delta_{0}-2 \delta_{1 / 2}+\delta_{1}$, where $\delta_{t}$ is the one-dimensional Dirac function at a point $t$. Hence for any univariate function $h \in C^{2}([0,1])$, we have

$$
\int_{0}^{1} h^{\prime \prime}(t) \min \{t, 1-t\} d t=h(0)-2 h(1 / 2)+h(1) .
$$

Combining this result with (2.14) we obtain (2.15).

\section{Positive Quadratic Functions}

In this section, we study the algorithm when applied to a quadratic polynomial $q$ such that $\operatorname{det}(\mathbf{q})>0$. We shall assume, without loss of generality, that $\mathbf{q}$ is positive definite, since all our results extend in a trivial manner to the negative definite case.

Our first observation is that the refinement procedure based on the decision function (2.13) always selects for bisection the longest edge in the sense of the q-metric $|\cdot|_{\mathbf{q}}$ defined by (2.2).

Lemma 3.1. An edge e of $T$ maximizes $D_{T}(e, q)$ among all edges of $T$ if and only if it maximizes $|e|_{\mathbf{q}}$ among all edges of $T$.

Proof. The hessian $d^{2} q$ is constant and for all $e \in \mathbb{R}^{2}$ one has

$$
\left\langle d^{2} q e, e\right\rangle=2 \mathbf{q}(e) .
$$

If $e$ is an edge of a triangle $T$, and if $q$ is a convex quadratic function, equation (2.15) therefore gives

$$
D_{T}(e, q)=\frac{|T|}{3} \mathbf{q}(e) \int_{0}^{1} \min \{t, 1-t\} d t=\frac{|T|}{12}|e|_{\mathbf{q}}^{2} .
$$

This concludes the proof.

It follows from this lemma that the longest edge of $T$ in the sense of the $\mathbf{q}$-metric is selected for bisection by the decision function. In the remainder of this section, we use this fact to prove that the refinement procedure produces triangles which tend to adopt an optimal aspect ratio in the sense that $\rho_{\mathbf{q}}(T)$ becomes small in an average sense.

For this purpose, it is convenient to introduce a close variant to $\rho_{\mathbf{q}}(T)$ : if $T$ is a triangle with edges $a, b, c$, such that $|a|_{\mathbf{q}} \geq|b|_{\mathbf{q}} \geq|c|_{\mathbf{q}}$, we define

$$
\sigma_{\mathbf{q}}(T):=\frac{\mathbf{q}(b)+\mathbf{q}(c)}{4|T| \sqrt{\operatorname{det} \mathbf{q}}}=\frac{|b|_{\mathbf{q}}^{2}+|c|_{\mathbf{q}}^{2}}{4|T| \sqrt{\operatorname{det} \mathbf{q}}} .
$$

Using the inequalities $|b|_{\mathbf{q}}^{2}+|c|_{\mathbf{q}}^{2} \leq 2|a|_{\mathbf{q}}^{2}$ and $|a|_{\mathbf{q}}^{2} \leq 2\left(|b|_{\mathbf{q}}^{2}+|c|_{\mathbf{q}}^{2}\right)$, we obtain the equivalence

$$
\frac{\rho_{\mathbf{q}}(T)}{8} \leq \sigma_{\mathbf{q}}(T) \leq \frac{\rho_{\mathbf{q}}(T)}{2} .
$$

Similar to $\rho_{\mathbf{q}}$, this quantity is invariant under a linear coordinate change $\phi$, in the sense that

$$
\sigma_{\mathbf{q} \circ \phi}(T)=\sigma_{\mathbf{q}}(\phi(T)),
$$

From (2.10) and (3.3) we can relate $\sigma_{\mathbf{q}}$ to the local approximation error. 
Proposition 3.2. There exists a constant $C_{0}$, which depends only on the choice of $\mathcal{A}_{T}$, such that for any triangle $T$, quadratic function $q$ and exponent $1 \leq p \leq \infty$, the local $L^{p}$-approximation error satisfies

$$
C_{0}^{-1} e_{T}(q)_{p} \leq \sigma_{\mathbf{q}}(T)\|\sqrt{\operatorname{det} \mathbf{q}}\|_{L^{\tau}(T)} \leq C_{0} e_{T}(q)_{p},
$$

where $\frac{1}{\tau}:=\frac{1}{p}+1$.

Our next result shows that $\sigma_{\mathbf{q}}(T)$ is always reduced by the refinement procedure.

Proposition 3.3. If $T$ is a triangle with children $T_{1}$ and $T_{2}$ is obtained by the refinement procedure for the quadratic function $q$, then

$$
\max \left\{\sigma_{\mathbf{q}}\left(T_{1}\right), \sigma_{\mathbf{q}}\left(T_{2}\right)\right\} \leq \sigma_{\mathbf{q}}(T) .
$$

Proof. Assuming that $|a|_{\mathbf{q}} \geq|b|_{\mathbf{q}} \geq|c|_{\mathbf{q}}$, we know that the edge $a$ is cut and that the children have area $|T| / 2$ and edges $a / 2, b,(c-b) / 2$ and $a / 2,(b-c) / 2, c$ (recall that $a+b+c=0)$. We then have

$$
\begin{aligned}
2|T| \sqrt{\operatorname{det} \mathbf{q}} \sigma_{\mathbf{q}}\left(T_{i}\right) & \leq \mathbf{q}\left(\frac{a}{2}\right)+\mathbf{q}\left(\frac{b-c}{2}\right) \\
& =\mathbf{q}\left(\frac{b+c}{2}\right)+\mathbf{q}\left(\frac{b-c}{2}\right) \\
& =\frac{\mathbf{q}(b)+\mathbf{q}(c)}{2} \\
& =2|T| \sqrt{\operatorname{det} \mathbf{q}} \sigma_{\mathbf{q}}(T) .
\end{aligned}
$$

Remark 3.4. When $\mathbf{q}$ is the euclidean metric, the triangle that minimizes $\sigma_{\mathbf{q}}$ is the half square. This is consistent with the above result since it is the only triangle which is similar (i.e., identical up to a translation, a rotation and a dilation) to both of its children after one step of longest edge bisection.

Remark 3.5. A result of similar nature was already proved in [11: longest edge bisection has the effect that the minimal angle in any triangle after an arbitrary number of refinements is at most twice the minimal angle of the initial triangle.

Our next objective is to show that as we iterate the refinement process, the value of $\sigma_{\mathbf{q}}(T)$ becomes bounded independently of $q$ for almost all generated triangles. For this purpose we introduce the following notation: if $T$ is a triangle with edges such that $|a|_{\mathbf{q}} \geq|b|_{\mathbf{q}} \geq|c|_{\mathbf{q}}$, we denote by $\psi_{\mathbf{q}}(T)$ the subtriangle of $T$ obtained after a bisection of $a$ which contains the smallest edge $c$. We first establish inequalities between the measures $\sigma_{\mathbf{q}}$ and $\rho_{\mathbf{q}}$ applied to $T$ and $\psi_{\mathbf{q}}(T)$.

Proposition 3.6. Let $T$ be a triangle, then

$$
\begin{aligned}
\sigma_{\mathbf{q}}\left(\psi_{\mathbf{q}}(T)\right) & \leq \frac{5}{8} \rho_{\mathbf{q}}(T), \\
\rho_{\mathbf{q}}\left(\psi_{\mathbf{q}}(T)\right) & \leq \frac{\rho_{\mathbf{q}}(T)}{2}\left(1+\frac{16}{\rho_{\mathbf{q}}^{2}(T)}\right) .
\end{aligned}
$$

Proof. We first prove (3.8). Obviously, $\psi_{\mathbf{q}}(T)$ contains one edge $s \in\{a, b, c\}$ from $T$, and one half edge $t \in\left\{\frac{a}{2}, \frac{b}{2}, \frac{c}{2}\right\}$ from $T$. Therefore,

$$
\sigma_{\mathbf{q}}\left(\psi_{\mathbf{q}}(T)\right) \leq \frac{|s|_{\mathbf{q}}^{2}+|t|_{\mathbf{q}}^{2}}{4\left|\psi_{\mathbf{q}}(T)\right| \sqrt{\operatorname{det} \mathbf{q}}} \leq \frac{|a|_{\mathbf{q}}^{2}+\left|\frac{a}{2}\right|_{\mathbf{q}}^{2}}{2|T| \sqrt{\operatorname{det} \mathbf{q}}}=\frac{5}{8} \rho_{\mathbf{q}}(T)
$$


For the proof of (3.9), we restrict our attention to the case $\mathbf{q}=x^{2}+y^{2}$, without loss of generality, thanks to the invariance formula (2.9). Let $T$ be a triangle with edges $|a| \geq|b| \geq|c|$. If $h$ is the width of $T$ in the direction perpendicular to $a$, then

$$
h=\frac{2|T|}{|a|}=\frac{2|a|}{\rho_{\mathbf{q}}(T)} .
$$

The sub-triangle $\psi_{\mathbf{q}}(T)$ of $T$ has edges $\frac{a}{2}, c, d$ where $d=\frac{b-c}{2}$, and the angles at the ends of $\frac{a}{2}$ are acute. Indeed,

$$
\langle c, a / 2\rangle=\frac{1}{4}\left(|b|^{2}-|a|^{2}-|c|^{2}\right) \leq 0 \text { and }\langle d, a / 2\rangle=\frac{1}{4}\left(|c|^{2}-|b|^{2}\right) \leq 0 .
$$

By Pythagora's theorem we thus find

$$
\max \left\{\left|\frac{a}{2}\right|^{2},|c|^{2},|d|^{2}\right\} \leq\left|\frac{a}{2}\right|^{2}+h^{2}=\frac{|a|^{2}}{4}\left(1+\frac{16}{\rho_{\mathbf{q}}^{2}(T)}\right) .
$$

Dividing by the respective areas of $T$ and $\psi_{\mathbf{q}}(T)$, we obtain the announced result. $\diamond$

Our next result shows that a significant reduction of $\sigma_{\mathbf{q}}$ occurs at least for one of the triangles obtained by three successive refinements, unless it has reached a small value of $\sigma_{\mathbf{q}}$. We use the notation $\psi_{\mathbf{q}}^{2}(T):=\psi_{\mathbf{q}}\left(\psi_{\mathbf{q}}(T)\right)$ and $\psi_{\mathbf{q}}^{3}(T):=\psi_{\mathbf{q}}\left(\psi_{\mathbf{q}}^{2}(T)\right)$.

Proposition 3.7. Let $T$ be a triangle such that $\sigma_{\mathbf{q}}\left(\psi_{\mathbf{q}}^{3}(T)\right) \geq 5$. Then $\sigma_{\mathbf{q}}\left(\psi_{\mathbf{q}}^{3}(T)\right) \leq$ $0.69 \sigma_{\mathbf{q}}(T)$.

Proof. The monotonicity of $\sigma_{\mathbf{q}}$ established in Proposition (3.3) implies that

$$
5 \leq \sigma_{\mathbf{q}}\left(\psi_{\mathbf{q}}^{3}(T)\right) \leq \sigma_{\mathbf{q}}\left(\psi_{\mathbf{q}}^{2}(T)\right) \leq \sigma_{\mathbf{q}}\left(\psi_{\mathbf{q}}(T)\right) .
$$

Combining this with inequality (3.8) we obtain

$$
8 \leq \min \left\{\rho_{\mathbf{q}}\left(\psi_{\mathbf{q}}^{2}(T)\right), \rho_{\mathbf{q}}\left(\psi_{\mathbf{q}}(T)\right), \rho_{\mathbf{q}}(T)\right\} .
$$

According to inequality (3.9), if a triangle $S$ obeys $\rho_{\mathbf{q}}(S) \geq 4$, then $\frac{1}{2}\left(1+\frac{16}{\rho_{\mathbf{q}}^{2}(S)}\right) \leq$ 1 and therefore $\rho_{\mathbf{q}}\left(\psi_{\mathbf{q}}(S)\right) \leq \rho_{\mathbf{q}}(S)$. We can apply this to $S=\psi_{\mathbf{q}}(T)$ and $S=T$, therefore obtaining

$$
\rho_{\mathbf{q}}\left(\psi_{\mathbf{q}}^{2}(T)\right) \leq \rho_{\mathbf{q}}\left(\psi_{\mathbf{q}}(T)\right) \leq \rho_{\mathbf{q}}(T) .
$$

We now remark that inequality (3.9) is equivalent to $\left(\rho_{\mathbf{q}}(S)-\rho_{\mathbf{q}}\left(\psi_{\mathbf{q}}(S)\right)\right)^{2} \geq$ $\rho_{\mathbf{q}}\left(\psi_{\mathbf{q}}(S)\right)^{2}-16$, hence

$$
\rho_{\mathbf{q}}(S) \geq \rho_{\mathbf{q}}\left(\psi_{\mathbf{q}}(S)\right)+\sqrt{\rho_{\mathbf{q}}\left(\psi_{\mathbf{q}}(S)\right)^{2}-16}
$$

provided that $\rho_{\mathbf{q}}(S) \geq \rho_{\mathbf{q}}\left(\psi_{\mathbf{q}}(S)\right)$. Apply this to $S=\psi_{\mathbf{q}}(T)$ and recalling that $\rho_{\mathbf{q}}\left(\psi_{\mathbf{q}}^{2}(T)\right) \geq 8$ we obtain

$$
\rho_{\mathbf{q}}\left(\psi_{\mathbf{q}}(T)\right) \geq 8+\sqrt{8^{2}-16} \geq 14.9 .
$$

Applying again (3.11) to $S=T$ we obtain

$$
\rho_{\mathbf{q}}(T) \geq 14.9+\sqrt{14.9^{2}-16} \geq 29.3 .
$$

Using (3.9), it follows that

$$
\frac{\rho\left(\psi_{\mathbf{q}}^{3}(T)\right)}{\rho(T)} \leq \frac{1}{8}\left(1+\frac{16}{\rho_{\mathbf{q}}^{2}\left(\psi_{\mathbf{q}}^{2}(T)\right)}\right)\left(1+\frac{16}{\rho_{\mathbf{q}}^{2}\left(\psi_{\mathbf{q}}(T)\right)}\right)\left(1+\frac{16}{\rho_{\mathbf{q}}^{2}(T)}\right) \leq 0.171 .
$$


Eventually, the inequalities (3.3) imply that

$$
2 \sigma_{\mathbf{q}}\left(\psi_{\mathbf{q}}^{3}(T)\right) \leq \rho_{\mathbf{q}}\left(\psi_{\mathbf{q}}^{3}(T)\right) \leq 0.171 \rho_{\mathbf{q}}(T) \leq 0.171\left(8 \sigma_{\mathbf{q}}(T)\right)
$$

which concludes the proof.

An immediate consequence of Propositions 3.3 and 3.7 is the following.

Corollary 3.8. If $\left(T_{i}\right)_{i=1}^{8}$ are the eight children obtained from three successive refinement procedures from $T$ for the function $q$, then

- for all $i, \sigma_{\mathbf{q}}\left(T_{i}\right) \leq \sigma_{\mathbf{q}}(T)$,

- there exists $i$ such that $\sigma_{\mathbf{q}}\left(T_{i}\right) \leq 0.69 \sigma_{\mathbf{q}}(T)$ or $\sigma_{\mathbf{q}}\left(T_{i}\right) \leq 5$.

We are now ready to prove that most triangles tend to adopt an optimal aspect ratio as one iterates the refinement procedure.

Theorem 3.9. Let $T$ be a triangle, and $\mathbf{q}$ a positive definite quadratic function. Let $k=\frac{\ln \sigma_{\mathbf{q}}(T)-\ln 5}{-\ln (0.69)}$. Then after $n$ applications of the refinement procedure starting from $T$, at most $C n^{k} 7^{n / 3}$ of the $2^{n}$ generated triangles satisfy $\sigma_{\mathbf{q}}(S) \geq 5$, where $C$ is an absolute constant. Therefore, the proportion of such triangles tends exponentially fast to 0 as $n \rightarrow+\infty$.

Proof. If we prove the proposition for $n$ multiple of 3 , then it will hold for all $n$ (with a larger constant) since $\sigma_{\mathbf{q}}$ decreases at each refinement step. We now assume that $n=3 m$, and consider the octree with root $T$ obtained by only considering the triangles of generation $3 i$ for $i=0, \ldots, n$.

According to Corollary 3.8, for each node of this tree, one of its eight children either checks $\sigma_{\mathbf{q}} \leq 5$ or has its non-degeneracy measure diminished by a factor $\theta:=0.69$. We remark that if $\sigma_{\mathbf{q}}$ is diminished at least $k$ times on the path going from the root $T$ to a leaf $S$, then $\sigma_{\mathbf{q}}(S) \leq 5$. As a consequence, the number $N(m)$ of triangles $S$ which are such that $\sigma_{\mathbf{q}}(S)>5$ within the generation level $n=3 m$ is bounded by the number of words in an eight letters alphabet $\left\{a_{1}, \ldots, a_{8}\right\}$ with length $m$ and which use the letter $a_{8}$ at most $k$ times, namely

$$
N(m) \leq \sum_{l=0}^{k}\left(\begin{array}{c}
m \\
l
\end{array}\right) 7^{m-l} \leq C m^{k} 7^{m}
$$

which is the announced result.

The fact that most triangles tend to adopt an optimal aspect ratio as one iterates the refinement procedure is a first hint that the approximation error in the greedy algorithm might satisfy the estimate (1.1) corresponding to an optimal triangulation. The following result shows that this is indeed the case, when this algorithm is applied on a triangular domain $\Omega$ to a quadratic function $q$ with positive definite associated quadratic form $\mathbf{q}$. The extension of this result to more general $C^{2}$ convex functions on polygonal domains requires a more involved analysis based on local perturbation arguments and is the object of the next section.

Corollary 3.10. Let $\Omega$ be a triangle, and $q$ a quadratic function with positive definite associated quadratic form $\mathbf{q}$. Let $q_{N}$ be the approximant of $q$ on $\Omega$ obtained by the greedy algorithm for the $L^{p}$ metric, using the $L^{1}$ decision function (2.13). Then

$$
\limsup _{N \rightarrow \infty} N\left\|q-q_{N}\right\|_{L^{p}(\Omega)} \leq C\|\sqrt{\operatorname{det}(\mathbf{q})}\|_{L^{\tau}(\Omega)},
$$


where $\frac{1}{\tau}=\frac{1}{p}+1$ and where the constant $C$ depends only on on the choice of the approximation operator $\mathcal{A}_{T}$ used in the definition of the approximant.

Proof. For any triangle $T$, quadratic function $q \in \Pi_{2}$, and exponent $p$, let

$$
e_{T}^{\prime}(q)_{p}:=\inf _{\pi \in \Pi_{1}}\|q-\pi\|_{L^{p}(T)}
$$

be the error of best approximation of $q$ on $T$. Let $T_{0}$ be a fixed triangle of area 1 , then for any $q \in \Pi_{2}$ and $1 \leq p \leq \infty$ one has

$$
e_{T_{0}}^{\prime}(q)_{1} \leq e_{T_{0}}^{\prime}(q)_{p} \leq e_{T_{0}}(q)_{p} \leq e_{T_{0}}(q)_{\infty}
$$

Furthermore, $e_{T_{0}}^{\prime}(\cdot)_{1}$ and $e_{T_{0}}(\cdot)_{\infty}$ are semi-norms on the finite dimensional space $\Pi_{2}$ which vanish precisely on the same subspace of $\Pi_{2}$, namely $\Pi_{1}$. Hence these semi-norms are equivalent. It follows that

$$
c_{0} e_{T_{0}}(q)_{p} \leq e_{T_{0}}^{\prime}(q)_{p} \leq e_{T_{0}}(q)_{p}
$$

where $c_{0}$ is independent $q \in \Pi_{2}$ and of $p \geq 1$. Using the invariance property (2.1) we find that (3.12) holds for any triangle $T$ in place of $T_{0}$ with the same constant $c_{0}$. We also define for any triangulation $\mathcal{T}$,

$$
e_{\mathcal{T}}(f)_{p}^{p}:=\sum_{T \in \mathcal{T}} e_{T}(f)_{p}^{p} \quad \text { and } \quad e_{\mathcal{T}}^{\prime}(f)_{p}^{p}:=\sum_{T \in \mathcal{T}} e_{T}^{\prime}(f)_{p}^{p}
$$

and we remark that $c_{0} e_{\mathcal{T}}(q)_{p} \leq e_{\mathcal{T}}^{\prime}(q)_{p} \leq e_{\mathcal{T}}(q)_{p}$. For each $n$, we denote by $\mathcal{T}_{n}^{u}$ the triangulation of $\Omega$ produced by $n$ successive refinements based on the $L^{1}$ decision function (2.13) for the quadratic function $q$ of interest (note that $\#\left(\mathcal{T}_{n}^{u}\right)=2^{n}$ ). We also define $\mathcal{T}_{n}^{\sigma}:=\left\{T \in \mathcal{T}_{n}^{u} ; \sigma_{\mathbf{q}}(T)>5\right\}$. Therefore $\sigma_{\mathbf{q}}(T) \leq 5$ if $T \notin \mathcal{T}_{n}^{\sigma}$, and on the other hand, we know from Proposition 3.3 that $\sigma_{\mathbf{q}}(T) \leq \sigma_{\mathbf{q}}(\Omega)$ for any $T \in \mathcal{T}_{n}^{u}$. It follows from Proposition 3.2 that

$$
\begin{aligned}
e_{\mathcal{T}_{n}^{u}}(q)_{p} & \leq C_{0}\left(\sum_{T \in \mathcal{T}_{n}^{u}}\left(\sigma_{\mathbf{q}}(T)|T|^{\frac{1}{\tau}} \sqrt{\operatorname{det} \mathbf{q}}\right)^{p}\right)^{\frac{1}{p}} \\
& \leq C_{0}\left(5^{p} \times 2^{n}+\sigma_{\mathbf{q}}(\Omega)^{p} \#\left(\mathcal{T}_{n}^{\sigma}\right)\right)^{\frac{1}{p}}\left(\frac{|\Omega|}{2^{n}}\right)^{\frac{1}{\tau}} \sqrt{\operatorname{det} \mathbf{q}}
\end{aligned}
$$

where $C_{0}$ is the constant in (3.4). According to Theorem 3.9, we know that

$$
\lim _{n \rightarrow+\infty} 2^{-n} \#\left(\mathcal{T}_{n}^{\sigma}\right)=0 \text {. }
$$

Hence

$$
\limsup _{n \rightarrow \infty} 2^{n} e_{\mathcal{T}_{n}^{u}}(q)_{p} \leq 5 C_{0}|\Omega|^{\frac{1}{\tau}} \sqrt{\operatorname{det} \mathbf{q}}=5 C_{0}\|\sqrt{\operatorname{det} \mathbf{q}}\|_{L^{\tau}(\Omega)}
$$

We now denote by $\mathcal{T}_{n}^{g}$ the triangulation generated by the greedy procedure with stopping criterion based on the error $\eta_{n}:=C_{0}^{-1} 2^{-\frac{n}{\tau}}\|\sqrt{\operatorname{det} \mathbf{q}}\|_{L^{\tau}(\Omega)}$. It follows from (3.4) that for all $T \in \mathcal{T}_{k}$ with $k \leq n$, one has

$$
e_{T}(q)_{p} \geq C_{0}^{-1} \sigma_{\mathbf{q}}(T)\|\sqrt{\operatorname{det} \mathbf{q}}\|_{L^{\tau}(T)} \geq C_{0}^{-1}\|\sqrt{\operatorname{det} \mathbf{q}}\|_{L^{\tau}(T)} \geq \eta_{n} .
$$

This shows that $\mathcal{T}_{g}^{n}$ is a refinement of $\mathcal{T}_{n}^{u}$. Furthermore, any triangle $T \in \mathcal{T}_{n}^{u}$ has at most $2^{k(T)}$ children in $\mathcal{T}_{n}^{g}$, where $k(T)$ is the smallest integer such that

$$
\eta_{n} \geq C_{0} 2^{-\frac{n+k(T)}{\tau}} \sigma_{\mathbf{q}}(T)\|\sqrt{\operatorname{det} \mathbf{q}}\|_{L^{\tau}(\Omega)} .
$$

Since $\frac{1}{2} \leq \tau \leq 1$ we obtain $2^{k(T)} \leq 2^{\frac{k(T)}{\tau}} \leq 2^{\frac{1}{\tau}} C_{0}^{2} \sigma_{q}(T) \leq 4 C_{0}^{2} \sigma_{q}(T)$. Hence

$$
\#\left(\mathcal{T}_{n}^{g}\right) \leq 4 C_{0}^{2} \sum_{T \in \mathcal{T}_{n}^{u}} \sigma_{q}(T) \leq 4 C_{0}^{2}\left(5 \times 2^{n}+\sigma_{\mathbf{q}}(\Omega) \#\left(\mathcal{T}_{n}^{\sigma}\right)\right)=C_{1} 2^{n}\left(1+\varepsilon_{n}\right),
$$


where $C_{1}=20 C_{0}^{2}$ and $\varepsilon_{n} \rightarrow 0$ as $n \rightarrow \infty$. If $\mathcal{T}_{N}$ is the triangulation generated after $N$ steps of the greedy algorithm, then there exists $n \geq 0$ such that $\mathcal{T}_{N}$ is a refinement of $\mathcal{T}_{n}^{g}$ (hence a refinement of $\mathcal{T}_{n}^{u}$ ) and $\mathcal{T}_{n+1}^{g}$ is a refinement of $\mathcal{T}_{N}$. It follows that $\#\left(\mathcal{T}_{N}\right) \leq \#\left(\mathcal{T}_{n+1}^{g}\right) \leq C_{1} 2^{n}\left(1+\varepsilon_{n}\right)$, and

$$
c_{0} e_{\mathcal{T}_{N}}(q)_{p} \leq e_{\mathcal{T}_{N}}^{\prime}(q)_{p} \leq e_{\mathcal{T}_{n}^{u}}^{\prime}(q)_{p} \leq e_{\mathcal{T}_{n}{ }^{u}}(q)_{p},
$$

where we have used the fact that $e_{\mathcal{T}}^{\prime}(f)_{p} \leq e_{\tilde{\mathcal{T}}}^{\prime}(f)_{p}$ whenever $\mathcal{T}$ is a refinement of $\tilde{\mathcal{T}}$. Eventually,

$$
\limsup _{N \rightarrow \infty} N e_{\mathcal{T}_{N}}(q)_{p} \leq \limsup _{n \rightarrow \infty} \frac{C_{1}}{c_{0}} 2^{n}\left(1+\varepsilon_{n}\right) e_{\mathcal{T}_{n}{ }^{u}}(q) \leq \frac{5 C_{0} C_{1}}{c_{0}}\|\sqrt{\operatorname{det} q}\|_{L^{\tau}(\Omega)}
$$

which concludes the proof.

\section{The CASE OF StRiCTLY CONVEX FUnCTIONS}

The goal of this section is to prove that the approximation error in the greedy algorithm applied to a $C^{2}$ function $f$ satisfies the estimate (1.1) corresponding to an optimal triangulation. Our main result is so far limited to the case where $f$ is strictly convex.

Theorem 4.1. Let $f \in C^{2}(\Omega)$ be such that

$$
d^{2} f(x) \geq m I, \text { for all } x \in \Omega,
$$

for some arbitrary but fixed $m>0$ independent of $x$. Let $f_{N}$ be the approximant obtained by the greedy algorithm for the $L^{p}$ metric, using the $L^{1}$ decision function (2.13). Then

$$
\limsup _{N \rightarrow \infty} N\left\|f-f_{N}\right\|_{L^{p}} \leq C\left\|\sqrt{\operatorname{det}\left(d^{2} f\right)}\right\|_{L^{\tau}}
$$

where $\frac{1}{\tau}=\frac{1}{p}+1$ and where $C$ is an absolute constant (i.e., independent of $p, f$ and $\alpha)$.

Equation (4.1) can be rephrased as follows: there exists a sequence $\varepsilon_{N}(f)$ such that $\varepsilon_{N} \rightarrow 0$ and

$$
\left\|f-f_{N}\right\|_{L^{p}} \leq\left(C\left\|\sqrt{\operatorname{det}\left(d^{2} f\right)}\right\|_{L^{\tau}}+\varepsilon_{N}(f)\right) N^{-1} .
$$

Note also that since $\left\|\sqrt{\operatorname{det}\left(d^{2} f\right)}\right\|_{L^{\tau}}>0$, there exists $N_{0}(f)$ such that $\left\|f-f_{N}\right\|_{L^{p}} \leq$ $2 C\left\|\sqrt{\operatorname{det}\left(d^{2} f\right)}\right\|_{L^{\tau}} N^{-1}$ for all $N \geq N_{0}(f)$. It should be stressed hard that $N_{0}(f)$ can be arbitrarily large depending on the function $f$. Intuitively, this means that when $f$ has a very large hessian at a certain point, it takes more iterations for the algorithm to generate triangles with a good aspect ratio. The extension of this result to strictly concave functions is immediate by a change of sign. Its extension to arbitrary $C^{2}$ functions is so far incomplete, as explained at the end of the introduction. The proof of Theorem 4.1 uses the fact that a strictly convex $C^{2}$ function is locally close to a quadratic function with positive definite hessian, which allows us to exploit the results obtained in $\S 3$ for these particular functions. 
4.1. A perturbation result. We consider a triangle $T$, a function $f \in C^{2}(T)$, a convex quadratic function $q$ and $\mu>0$ such that on $T$,

$$
d^{2} q \leq d^{2} f \leq(1+\mu) d^{2} q .
$$

It follows that $\operatorname{det}\left(d^{2} q\right) \leq \operatorname{det}\left(d^{2} f\right) \leq \operatorname{det}\left((1+\mu) d^{2} q\right)=(1+\mu)^{2} \operatorname{det}\left(d^{2} q\right)$. Since $\operatorname{det}\left(d^{2} q\right)=4 \operatorname{det}(\mathbf{q})$, we obtain

$$
2\|\sqrt{\operatorname{det} \mathbf{q}}\|_{L^{\tau}(T)} \leq\left\|\sqrt{\operatorname{det}\left(d^{2} f\right)}\right\|_{L^{\tau}(T)} \leq 2(1+\mu)\|\sqrt{\operatorname{det} \mathbf{q}}\|_{L^{\tau}(T)} .
$$

The following lemma shows how the local errors associated to $f$ and $q$ are close.

Proposition 4.2. The exists a constant $C_{e}>0$, depending only on the operator $\mathcal{A}_{T}$ such that

$$
\left(1-C_{e} \mu\right) e_{T}(q)_{p} \leq e_{T}(f)_{p} \leq\left(1+C_{e} \mu\right) e_{T}(q)_{p} .
$$

Proof. It follows from inequality (4.2) that the functions $f-q$ and $(1+\mu) q-f$ are convex, hence

$$
I_{T}(f-q)-(f-q) \geq 0 \text { and } I_{T}((1+\mu) q-f)-((1+\mu) q-f) \geq 0
$$

on the triangle $T$. We therefore obtain

$$
0 \leq\left(I_{T} f-f\right)-\left(I_{T} q-q\right) \leq \mu\left(I_{T} q-q\right) .
$$

There exists a constant $C_{0}>0$ depending only on $\mathcal{A}_{T}$ such that for any $h \in C^{0}(T)$,

$$
e_{T}(h)_{p} \leq C_{0}|T|^{\frac{1}{p}}\|h\|_{L^{\infty}(T)}
$$

Furthermore, according to Proposition 2.3 there exists a constant $C_{1}>0$ depending only on $\mathcal{A}_{T}$ such that

$$
|T|^{1 / p}\left\|q-I_{T} q\right\|_{L^{\infty}(T)} \leq C_{1} e_{T}(q)_{p} .
$$

Hence

$$
\begin{aligned}
\left|e_{T}(f)_{p}-e_{T}(q)_{p}\right| & \leq e_{T}(f-q)_{p} \\
& =e_{T}\left(\left(I_{T} f-f\right)-\left(I_{T} q-q\right)\right)_{p} \\
& \leq C_{0}|T|^{\frac{1}{p}}\left\|\left(I_{T} f-f\right)-\left(I_{T} q-q\right)\right\|_{L^{\infty}(T)} \\
& \leq C_{0}|T|^{\frac{1}{p}}\left\|\mu\left(I_{T} q-q\right)\right\|_{L^{\infty}(T)} \\
& \leq C_{0} C_{1} \mu e_{T}(q)_{p} .
\end{aligned}
$$

This concludes the proof of this lemma, with $C_{e}=C_{0} C_{1}$.

Note that using Proposition 3.2 and assuming that $\mu \leq c_{e}:=\frac{1}{2 C_{e}}$, we have with $\frac{1}{\tau}:=1+\frac{1}{p}$,

$$
e_{T}(f)_{p} \sim e_{T}(q)_{p} \sim \sigma_{\mathbf{q}}(T)\|\sqrt{|\operatorname{det} \mathbf{q}|}\|_{L^{\tau}(T)} \sim \sigma_{\mathbf{q}}(T)\left\|\sqrt{\operatorname{det}\left(d^{2} f\right)}\right\|_{L^{\tau}(T)},
$$

with absolute constants in the equivalence.

We next study the behavior of the decision function $e \mapsto d_{T}(e, f)$. For this purpose, we introduce the following definition.

Definition 4.3. Let $T$ be a triangle with edges $a, b, c$. A $\delta$-near longest edge bisection with respect to the q-metric is a bisection of any edge $e \in\{a, b, c\}$ such that

$$
\mathbf{q}(e) \geq(1-\delta) \max \{\mathbf{q}(a), \mathbf{q}(b), \mathbf{q}(c)\} .
$$


Proposition 4.4. Assume that $f$ and $q$ satisfy (4.2). Then, the bisection of $T$ prescribed by the decision function $e \mapsto d_{T}(e, f)$ is a $\mu$-near longest edge bisection for the $\mathbf{q}$-metric.

Proof. It follows directly from equation (2.15) that for any edge $e$ of $T$,

$$
D_{T}(e, q) \leq D_{T}(e, f) \leq D_{T}(e,(1+\mu) q),
$$

hence using (3.1) we obtain

$$
\frac{|T|}{12} \mathbf{q}(e) \leq D_{T}(e, f) \leq(1+\mu) \frac{|T|}{12} \mathbf{q}(e) .
$$

Therefore, the bisection of $T$ prescribed by the decision function $e \mapsto d_{T}(e, f)$ selects an $e$ such that

$$
(1+\mu) \mathbf{q}(e) \geq \max \{\mathbf{q}(a), \mathbf{q}(b), \mathbf{q}(c)\} .
$$

It is therefore a $\delta$-near longest edge bisection for the $\mathbf{q}$-metric with $\delta=\frac{\mu}{1+\mu} \leq \mu$ and therefore also a $\mu$-near longest edge bisection.

In the rest of this section, we analyze the difference between a longest edge bisection in the q-metric and a $\delta$-near longest edge bisection. For that purpose we introduce a distance between triangles: if $T_{1}, T_{2}$ are two triangles with edges $a_{1}, b_{1}, c_{1}$ and $a_{2}, b_{2}, c_{2}$ such that

$$
\mathbf{q}\left(a_{1}\right) \geq \mathbf{q}\left(b_{1}\right) \geq \mathbf{q}\left(c_{1}\right) \text { and } \mathbf{q}\left(a_{2}\right) \geq \mathbf{q}\left(b_{2}\right) \geq \mathbf{q}\left(c_{2}\right),
$$

we define

$$
\Delta_{\mathbf{q}}\left(T_{1}, T_{2}\right)=\max \left\{\left|\mathbf{q}\left(a_{1}\right)-\mathbf{q}\left(a_{2}\right)\right|,\left|\mathbf{q}\left(b_{1}\right)-\mathbf{q}\left(b_{2}\right)\right|,\left|\mathbf{q}\left(c_{1}\right)-\mathbf{q}\left(c_{2}\right)\right|\right\} .
$$

Note that $\Delta_{\mathbf{q}}$ is a distance up to rigid transformations.

Lemma 4.5. Let $T_{1}, T_{2}$ be two triangles, let $\left(R_{1}, U_{1}\right)$ and $\left(R_{2}, U_{2}\right)$ be the two pairs of children from the longest edge bisection of $T_{1}$ in the $\mathbf{q}$-metric, and a $\delta$-near longest edge bisection of $T_{2}$ in the $\mathbf{q}$-metric. Then, up to a permutation of the pair of triangles $\left(R_{1}, U_{1}\right)$,

$$
\max \left\{\Delta_{\mathbf{q}}\left(R_{1}, R_{2}\right), \Delta_{\mathbf{q}}\left(U_{1}, U_{2}\right)\right\} \leq \frac{5}{4} \Delta_{\mathbf{q}}\left(T_{1}, T_{2}\right)+\delta \mathbf{q}\left(a_{2}\right),
$$

where $a_{2}$ is the longest edge of $T_{2}$ in the $\mathbf{q}$-metric.

Proof. We assume that the edges of $T_{1}$ and $T_{2}$ are named and ordered as in (4.7). Up to a permutation, $R_{1}$ and $U_{1}$ have edge vectors $b_{1}, a_{1} / 2,\left(c_{1}-b_{1}\right) / 2$ and $c_{1}, a_{1} / 2,\left(b_{1}-\right.$ $\left.c_{1}\right) / 2$. Two situations might occur for the pair $\left(R_{2}, U_{2}\right)$ :

- $\mathbf{q}(e)<(1-\delta) \mathbf{q}\left(a_{2}\right)$ for $e=b_{2}$ and $c_{2}$. In such a case the triangle $T_{2}$ is bisected towards $a_{2}$, so that up to a permutation, $R_{2}$ and $U_{2}$ have edge vectors $b_{2}, a_{2} / 2,\left(c_{2}-b_{2}\right) / 2$ and $c_{2}, a_{2} / 2,\left(b_{2}-c_{2}\right) / 2$. Using that $q((c-$ b) $/ 2)=q(c) / 2+q(b) / 2-q(a) / 4$ when $a+b+c=0$, it clearly follows that

$$
\max \left\{\Delta_{\mathbf{q}}\left(R_{1}, R_{2}\right), \Delta_{\mathbf{q}}\left(U_{1}, U_{2}\right)\right\} \leq \frac{5}{4} \Delta_{\mathbf{q}}\left(T_{1}, T_{2}\right) .
$$

- $\mathbf{q}(e) \geq(1-\delta) \mathbf{q}\left(a_{2}\right)$ for some $e=b_{2}$ or $c_{2}$. In such a case $T_{2}$ may be bisected, say, towards $b_{2}$, so that up to a permutation, $R_{2}$ and $U_{2}$ have edge vectors $a_{2}, b_{2} / 2,\left(c_{2}-a_{2}\right) / 2$ and $c_{2}, b_{2} / 2,\left(b_{2}-c_{2}\right) / 2$. But since $\left|\mathbf{q}\left(b_{2}\right)-\mathbf{q}\left(a_{2}\right)\right|_{\mathbf{q}} \leq$ $\delta \mathbf{q}\left(a_{2}\right)$, we obtain that

$$
\max \left\{\Delta_{\mathbf{q}}\left(R_{1}, R_{2}\right), \Delta_{\mathbf{q}}\left(U_{1}, U_{2}\right)\right\} \leq \frac{5}{4} \Delta_{\mathbf{q}}\left(T_{1}, T_{2}\right)+\delta \mathbf{q}\left(a_{2}\right) .
$$


We now introduce a perturbed version of the estimates describing the decay of the non-degeneracy measure which were obtained in Proposition 3.3 and Corollary 3.8 .

Proposition 4.6. If $\left(T_{i}\right)_{i=1}^{2}$ are the two children obtained from a refinement of a triangle $T$ in which a $\delta$-near longest edge bisection in the $\mathbf{q}$-metric is selected, then

$$
\max \left\{\sigma_{\mathbf{q}}\left(T_{1}\right), \sigma_{\mathbf{q}}\left(T_{2}\right)\right\} \leq(1+4 \delta) \sigma_{\mathbf{q}}(T) .
$$

If $\left(T_{i}\right)_{i=1}^{8}$ are the eight children of a triangle $T$ obtained from three successive refinements in which a $\delta$-near longest edge bisection in the $\mathbf{q}$-metric is selected, then

- for all $i, \sigma_{\mathbf{q}}\left(T_{i}\right) \leq \sigma_{\mathbf{q}}(T)\left(1+C_{2} \delta\right)$,

- there exists $i$ such that $\sigma_{\mathbf{q}}\left(T_{i}\right) \leq 0.69 \sigma_{\mathbf{q}}(T)\left(1+C_{2} \delta\right)$ or $\sigma_{\mathbf{q}}\left(T_{i}\right) \leq M$, where $C_{2}=\frac{61}{4}$ and $M=5\left(1+C_{2} \delta\right)$.

Proof. We first prove (4.9), and for that purpose we introduce the two children $T_{1}^{\prime}, T_{2}^{\prime}$ obtained by bisecting the longest edge of $T$ in the $\mathbf{q}$-metric. It follows from (4.8) that, up to a permutation of the pair $\left(T_{1}^{\prime}, T_{2}^{\prime}\right)$,

$$
\max \left\{\Delta_{\mathbf{q}}\left(T_{1}, T_{1}^{\prime}\right), \Delta_{\mathbf{q}}\left(T_{2}, T_{2}^{\prime}\right)\right\} \leq \delta \mathbf{q}(a),
$$

where $a$ is the longest edge of $T$ in the q-metric. Hence

$$
\left|\sigma_{\mathbf{q}}\left(T_{i}\right)-\sigma_{\mathbf{q}}\left(T_{i}^{\prime}\right)\right| \leq \frac{2 \Delta_{\mathbf{q}}\left(T_{i}, T_{i}^{\prime}\right)}{4\left|T_{i}\right| \sqrt{\operatorname{det}(\mathbf{q})}} \leq 2 \delta \frac{\mathbf{q}(a)}{4\left|T_{i}\right| \sqrt{\operatorname{det}(\mathbf{q})}} \leq 4 \delta \sigma_{\mathbf{q}}(T) .
$$

We know from Proposition 3.3 that $\max \left\{\sigma_{\mathbf{q}}\left(T_{1}^{\prime}\right), \sigma_{\mathbf{q}}\left(T_{2}^{\prime}\right)\right\} \leq \sigma_{\mathbf{q}}(T)$. Combining this point with (4.10) we conclude the proof of (4.9).

We now turn to the proof of the second part of the proposition and for that purpose we introduce the eight children $\left(T_{i}^{\prime}\right)_{i=1}^{8}$ obtained from three successive refinements of $T$ in which the longest edge in the q-metric is selected. Iterating (4.8), we find that, up to a permutation of the triangles $\left(T_{i}^{\prime}\right)_{i=1}^{8}$, one has

$$
\max _{i=1, \cdots, 8} \Delta_{\mathbf{q}}\left(T_{i}, T_{i}^{\prime}\right) \leq\left(1+\frac{5}{4}+\left(\frac{5}{4}\right)^{2}\right) \delta \mathbf{q}(a)=\frac{61}{16} \delta \mathbf{q}(a)=\frac{C_{2} \delta}{4} q(a),
$$

where, again, $a$ is the longest edge of $T$ in the $\mathbf{q}$-metric. Repeating the argument (4.10) we find that

$$
\max _{i=1, \ldots, 8}\left|\sigma_{\mathbf{q}}\left(T_{i}\right)-\sigma_{\mathbf{q}}\left(T_{i}^{\prime}\right)\right| \leq C_{2} \delta \sigma_{\mathbf{q}}(T) .
$$

We know from Corollary 3.8 that $\sigma_{\mathbf{q}}\left(T_{i}^{\prime}\right) \leq \sigma_{\mathbf{q}}(T)$ for all $i$ and that there exists $i$ such that either $\sigma_{\mathbf{q}}\left(T_{i}^{\prime}\right) \leq 0.69 \sigma_{\mathbf{q}}(T)$ or $\sigma_{\mathbf{q}}\left(T_{i}^{\prime}\right) \leq 5$. Combining this point with (4.11) we conclude the proof of the proposition.

4.2. Local optimality. Our next step towards the proof of Theorem 4.1 is to show that the triangulation produced by the greedy algorithm is locally optimal in the following sense: if the refinement procedure for the function $f$ produces a triangle $T \in \mathcal{D}$ on which $f$ is close enough to a quadratic function $q$, then the triangles which are generated from the refinement of $T$ tend to adopt an optimal aspect ratio in the q-metric, and a local version of the optimal estimate (1.1) holds on $T$.

We first prove that most triangles adopt an optimal aspect ratio as we iterate the refinement procedure. Our goal is thus to obtain a result similar to Theorem 3.9 which was restricted to quadratic functions. However, due to the perturbations 
by $C_{2} \mu$ that appear in Proposition 4.6, the formulation will be slightly different, yet sufficient for our purposes: we shall prove that the measure of non-degeneracy becomes bounded by an absolute constant in an average sense, as we iterate the refinement procedure.

As in the previous section, we assume that $f$ and $q$ satisfy (4.2). For any $T$, we define $\mathcal{T}_{n}^{u}(T)$ the triangulation of $T$ which is built by iteratively applying the refinement procedure for the function $f$ to all generated triangles up to $3 n$ generation levels. Note that

$$
\#\left(\mathcal{T}_{n}^{u}(T)\right)=2^{3 n} \text { and }\left|T^{\prime}\right|=2^{-3 n}|T|, \quad T^{\prime} \in \mathcal{T}_{n}^{u}(T)
$$

For $r>0$, we define the average $r$-th power of the measure of non-degeneracy of the $2^{3 n}$ triangles obtained from $T$ after $3 n$ iterations by

$$
\overline{\sigma_{\mathbf{q}}^{r}(n)}=\frac{1}{2^{3 n}} \sum_{T^{\prime} \in \mathcal{T}_{n}^{u}(T)} \sigma_{\mathbf{q}}^{r}\left(T^{\prime}\right)
$$

We also define

$$
\gamma(r, \mu):=\frac{1}{8}\left(0.69\left(1+C_{2} \mu\right)\right)^{r}+\frac{7}{8}\left(1+C_{2} \mu\right)^{r}
$$

where $C_{2}$ is the constant in Proposition 4.6. Note that for any $r>0$, the function $\gamma(r, \cdot)$ is continuous and increasing, and that $0<\gamma(r, 0)<1$. Hence for any $r>0$, there exists $\mu(r)>0$ and $0<\gamma(r)<1$ such that $\gamma(r, \mu) \leq \gamma(r)$, if $0<\mu<\mu(r)$.

Proposition 4.7. Assume that $f$ and $q$ satisfy (4.2) with $0<\mu \leq \mu(r)$. We then have

$$
\overline{\sigma_{\mathbf{q}}^{r}(n)} \leq \sigma_{\mathbf{q}}^{r}(T) \gamma(r)^{n}+\frac{M^{r}}{8(1-\gamma(r))}
$$

where $M$ is the constant in Proposition 4.6. Therefore,

$$
\overline{\sigma_{\mathbf{q}}^{r}(n)} \leq C_{3}:=1+\frac{M^{r}}{8(1-\gamma(r))}
$$

if $2^{3 n} \geq 8 \sigma_{\mathbf{q}}(T)^{\lambda}$ with $\lambda:=\frac{3 r \ln 2}{-\ln \gamma(r)}$.

Proof. Let us use the notations $u=\frac{3}{4}\left(1+C_{2} \mu\right)$ and $v=\left(1+C_{2} \mu\right)$. According to Proposition 4.6, we have

$$
\overline{\sigma_{\mathbf{q}}^{r}(n)} \leq \mathbb{E}\left(\sigma_{n}^{r}\right)
$$

where $\mathbb{E}$ is the expectation operator and $\sigma_{n}$ is the Markov chain with value in $[1,+\infty[$ defined by

- $\sigma_{n+1}=\max \left\{\sigma_{n} u, M\right\}$ with probability $\alpha:=\frac{1}{8}$,

- $\sigma_{n+1}=\sigma_{n} v$ with probability $\beta:=\frac{7}{8}$,

- $\sigma_{0}:=\sigma_{\mathbf{q}}\left(T_{0}\right)$ with probability 1 . 
Denoting by $\mu_{n}$ the probability distribution of $\sigma_{n}$, we have

$$
\begin{aligned}
\mathbb{E}\left(\sigma_{n+1}^{r}\right) & =\int_{1}^{\infty} \sigma^{r} d \mu_{n+1}(\sigma) \\
& =\int_{1}^{\infty}\left(\alpha(\max \{u \sigma, M\})^{r}+\beta(v \sigma)^{r}\right) d \mu_{n}(\sigma) \\
& =\alpha M^{r} \int_{1}^{M / u} d \mu_{n}(\sigma)+\alpha u^{r} \int_{M / u}^{\infty} \sigma^{r} d \mu_{n}(\sigma)+\beta v^{r} \int_{1}^{+\infty} \sigma^{r} d \mu_{n}(\sigma) \\
& \leq \alpha M^{r}+\left(\alpha u^{r}+\beta v^{r}\right) \mathbb{E}\left(\sigma_{n}^{r}\right) \\
& \leq \alpha M^{r}+\gamma(r) \mathbb{E}\left(\sigma_{n}^{r}\right) .
\end{aligned}
$$

By iteration, it follows that

$$
\mathbb{E}\left(\sigma_{n}^{r}\right) \leq \mathbb{E}\left(\sigma_{0}^{r}\right) \gamma(r)^{n}+\frac{\alpha M^{r}}{1-\gamma(r)},
$$

which gives the result.

Our next goal is to show that the greedy algorithm initialized from $T$ generates a triangulation which is a refinement of $\mathcal{T}_{n}^{u}(T)$ and therefore more accurate, yet with a similar amount of triangles. To this end, we apply the greedy algorithm with root $T$ and stopping criterion given by the local error

$$
\eta:=\min _{T^{\prime} \in \mathcal{T}_{n}^{u}(T)} e_{T^{\prime}}(f)_{p}
$$

Therefore, $T^{\prime}$ is splitted if and only if $e_{T^{\prime}}(f)_{p}>\eta$. We denote by $\mathcal{T}_{N}(T)$ the resulting triangulation where $N$ is its cardinality. From the definition of the stopping criterion, it is clear that $\mathcal{T}_{N}(T)$ is a refinement of $\mathcal{T}_{n}^{u}(T)$.

Proposition 4.8. Assume that $f$ and $q$ satisfy (4.2) with $\mu \leq \frac{1}{8}$, and define $r_{0}:=$ $\frac{\ln 2}{\ln 4-\ln 3}>0$. We then have

$$
N \leq C_{4} 2^{3 n} \overline{\sigma_{\mathbf{q}}^{r_{0}}(n)},
$$

where $C_{4}$ is an absolute constant. Assuming, in addition, that $\mu \leq \mu\left(r_{0}\right)$ as in Proposition 4.7, we obtain that

$$
N \leq C_{5} 2^{3 n}
$$

if $2^{3 n} \geq 8 \sigma_{\mathbf{q}}(T)^{\lambda}$ with $\lambda:=\frac{3 r_{0} \ln 2}{-\ln \gamma\left(r_{0}\right)}$, and where $C_{5}=C_{3} C_{4}$.

Proof. Let $T_{1}$ be a triangle in $\mathcal{T}_{n}^{u}(T)$ and $T_{2}$ a triangle in $\mathcal{T}_{N}(T)$ such that $T_{2} \subset T_{1}$. We shall give a bound on the number of splits $k$ which were applied between $T_{1}$ and $T_{2}$, i.e., such that $\left|T_{2}\right|=2^{-k}\left|T_{1}\right|$. We first remark that according to Proposition 3.2 and (4.5), we have

$$
\eta \geq c \min _{T^{\prime} \in \mathcal{T}_{n}^{u}(T)}\left|T^{\prime}\right|^{1+\frac{1}{p}} \sigma_{\mathbf{q}}\left(T^{\prime}\right) \sqrt{\operatorname{det} \mathbf{q}} \geq c\left|T_{1}\right|^{1+\frac{1}{p}} \sqrt{\operatorname{det} \mathbf{q}}
$$


where $c$ is an absolute constant. On the other hand, using both Propositions 4.2 and 4.6, we obtain

$$
\begin{aligned}
e_{T_{2}}(f)_{\mathbf{q}} & \leq C\left|T_{2}\right|^{1+\frac{1}{p}} \sigma_{\mathbf{q}}\left(T_{2}\right) \sqrt{\operatorname{det} \mathbf{q}} \\
& =\left|T_{1}\right|^{1+\frac{1}{p}} 2^{-k\left(1+\frac{1}{p}\right)} \sigma_{\mathbf{q}}\left(T_{2}\right) \sqrt{\operatorname{det} \mathbf{q}} \\
& \leq C\left|T_{1}\right|^{1+\frac{1}{p}} \sigma_{\mathbf{q}}\left(T_{1}\right)\left(2^{-\left(1+\frac{1}{p}\right)}(1+4 \mu)\right)^{k} \sqrt{\operatorname{det} \mathbf{q}} \\
& \leq \frac{C}{c} \sigma_{\mathbf{q}}\left(T_{1}\right)\left(\frac{1+4 \mu}{2}\right)^{k} \eta \\
& \leq \frac{C}{c} \sigma_{\mathbf{q}}\left(T_{1}\right)\left(\frac{3}{4}\right)^{k} \eta
\end{aligned}
$$

where $C$ is an absolute constant. Therefore, we see that $k$ is at most the smallest integer such that $\frac{C}{c} \sigma_{\mathbf{q}}\left(T_{1}\right)\left(\frac{3}{4}\right)^{k} \leq 1$. It follows that the total number $n\left(T_{1}\right)$ of triangles $T_{2} \in \mathcal{T}_{N}(T)$ which are contained in $T_{1}$ is bounded by

and therefore

$$
n\left(T_{1}\right) \leq 2^{k} \leq 2\left(\frac{C}{c} \sigma_{\mathbf{q}}\left(T_{1}\right)\right)^{r_{0}},
$$

$$
N^{\prime}=\sum_{T_{1} \in \mathcal{T}_{n}^{u}(T)} n\left(T_{1}\right) \leq 2\left(\frac{C}{c}\right)^{r_{0}} \sum_{T_{1} \in \mathcal{T}_{n}^{u}(T)} \sigma_{\mathbf{q}}\left(T_{1}\right)^{r_{0}}=C_{4} 2^{3 n} \overline{\sigma_{\mathbf{q}}^{r_{0}}(n)},
$$

with $C_{4}=2\left(\frac{C}{c}\right)^{r_{0}}$. The fact that $N \leq C_{5} 2^{3 n}$ when $2^{3 n} \geq 8 \sigma_{\mathbf{q}}(T)^{\lambda}$ with $\lambda:=$ $\frac{3 r_{0} \ln 2}{-\ln \gamma\left(r_{0}\right)}$ is an immediate consequence of Proposition 4.7 ,

4.3. Optimal convergence estimates. Our last step towards the proof of Theorem 4.1 consists in deriving local error estimates for the greedy algorithm. For $\eta>0$, we denote by $f_{\eta}$ the approximant to $f$ obtained by the greedy algorithm with stopping criterion given by the local error $\eta$ : a triangle $T$ is split if and only if $e_{T}(f)_{p}>\eta$. The resulting triangulation is denoted by

$$
\mathcal{T}_{\eta}=\mathcal{T}_{N} \text { with } N=N(\eta)=\#\left(\mathcal{T}_{\eta}\right) .
$$

For this $N$, we thus have $f_{\eta}=f_{N}$. For a given $T$ generated by the refinement procedure such that $\eta \leq e_{T}(f)_{p}$, we also define

$$
\mathcal{T}_{\eta}(T)=\left\{T^{\prime} \subset T ; T^{\prime} \in \mathcal{T}_{\eta}\right\},
$$

the triangles in $\mathcal{T}_{\eta}$ which are contained in $T$ and

$$
N(T, \eta)=\#\left(\mathcal{T}_{\eta}(T)\right) \text {. }
$$

Our next result provides estimates of the local error $\left\|f-f_{\eta}\right\|_{L^{p}(T)}$ and of $N(T, \eta)$ in terms of $\eta$, provided that $\mu$ is small enough.

Theorem 4.9. Assume that $f$ and $q$ satisfy (4.2) with $\mu \leq c_{2}:=\min \left\{\frac{1}{8}, \mu\left(r_{0}\right)\right\}$, and that $\eta \leq \eta_{0}$, where

$$
\eta_{0}=\eta_{0}(T):=\left(\frac{|T|}{\sigma_{\mathbf{q}}(T)^{\lambda}}\right)^{\frac{1}{\tau}} \sqrt{\operatorname{det} \mathbf{q}}
$$

with $\lambda:=\frac{3 r_{0} \ln 2}{-\ln \gamma\left(r_{0}\right)}$ and $\frac{1}{\tau}=\frac{1}{p}+1$. Then

$$
\left\|f-f_{\eta}\right\|_{L^{p}(T)} \leq \eta N(T, \eta)^{\frac{1}{p}}
$$

and

$$
N(T, \eta) \leq C_{6} \eta^{-\tau}\left\|\sqrt{\operatorname{det}\left(d^{2} f\right)}\right\|_{L^{\tau}(T)}^{\tau},
$$


where $C_{6}$ is an absolute constant.

Proof. The first estimate is trivial since

$$
\left\|f-f_{\eta}\right\|_{L^{p}(T)}=\left(\sum_{T^{\prime} \in \mathcal{T}_{\eta}(T)} e_{T^{\prime}}(f)_{p}^{p}\right)^{\frac{1}{p}} \leq\left(\sum_{T^{\prime} \in \mathcal{T}_{\eta}(T)} \eta^{p}\right)^{\frac{1}{p}}=\eta N(T, \eta)^{\frac{1}{p}} .
$$

In the case $p=\infty$, we trivially have

$$
\left\|f-f_{\eta}\right\|_{L^{\infty}(T)} \leq \eta .
$$

For the second estimate, we define $n_{0}=n_{0}(T)$, the smallest positive integer such that $2^{3 n_{0}(T)} \geq 8 \sigma_{\mathbf{q}}(T)^{\lambda}$ with $\lambda:=\frac{3 r_{0} \ln 2}{-\ln \gamma\left(r_{0}\right)}$. For any fixed $n \geq n_{0}$, we define

$$
\eta_{n}:=\min _{T^{\prime} \in \mathcal{T}_{n}^{u}(T)} e_{T^{\prime}}(f)_{p} .
$$

We know from Proposition 4.8 that with the choice $\eta=\eta_{n}$,

$$
N\left(T, \eta_{n}\right) \leq C_{5} 2^{3 n}
$$

On the other hand, we know from Proposition 4.7 that $\overline{\sigma_{\mathbf{q}}^{r_{0}}(n)} \leq C_{3}$, from which it follows that

$$
\min _{T^{\prime} \in \mathcal{T}_{n}^{u}(T)} \sigma_{\mathbf{q}}\left(T^{\prime}\right) \leq C_{3}^{\frac{1}{r_{0}}} .
$$

According to Proposition 4.2. we also have

$$
\eta_{n} \leq C \min _{T^{\prime} \in \mathcal{T}_{n}^{u}(T)}\left|T^{\prime}\right|^{1+\frac{1}{p}} \sigma_{\mathbf{q}}\left(T^{\prime}\right) \sqrt{\operatorname{det} \mathbf{q}} \leq C_{3}^{\frac{1}{r_{0}}} C\left(\frac{|T|}{2^{3 n}}\right)^{\frac{1}{\tau}} \sqrt{\operatorname{det} \mathbf{q}},
$$

where $C$ is an absolute constant, which also reads

$$
2^{3 n} \leq C_{3}^{\frac{\tau}{r_{0}}} C^{\tau} \eta_{n}^{-\tau}|T| \sqrt{\operatorname{det} \mathbf{q}}^{\tau} .
$$

Combining this with (4.14), we have obtained the estimate

$$
N\left(T, \eta_{n}\right) \leq C_{5} C_{3}^{\frac{\tau}{r_{0}}} C^{\tau} \eta_{n}^{-\tau}|T| \sqrt{\operatorname{det} \mathbf{q}}^{\tau},
$$

which by Proposition 4.2 is equivalent to (4.13) with $\eta=\eta_{n}$. In order to obtain (4.13) for all arbitrary values of $\eta$, we write that $\eta_{n+1}<\eta \leq \eta_{n}$ for some $n \geq n_{0}$, then

$$
\begin{aligned}
N(T, \eta) & \leq N\left(T, \eta_{n+1}\right) \\
& \leq C_{5} 2^{3(n+1)} \\
& \leq 8 C_{5} C_{3}^{\frac{\tau}{r_{0}}} C^{\tau} \eta_{n}^{-\tau}|T| \sqrt{\operatorname{det} \mathbf{q}}{ }^{\tau} \\
& \leq 8 C_{5} C_{3}^{\frac{\tau}{r_{0}}} C^{\tau} \eta^{-\tau}|T| \sqrt{\operatorname{det} \mathbf{q}}
\end{aligned}
$$

which by Proposition 4.2 is equivalent to (4.13). In the case where $\eta \geq \eta_{n_{0}}$, we simply write

$$
\begin{aligned}
N(T, \eta) & \leq N\left(T, \eta_{n_{0}}\right) \\
& \leq C_{5} 2^{3 n_{0}} \\
& \leq 64 C_{5} \sigma_{\mathbf{q}}(T)^{\lambda} \\
& =64 C_{5} \eta_{0}^{-\tau}|T| \sqrt{\operatorname{det} \mathbf{q}} \\
& \tau \\
& \leq 64 C_{5} \eta^{-\tau}|T| \sqrt{\operatorname{det} \mathbf{q}}
\end{aligned}
$$

and we conclude in the same way. 
We remark that combining the estimates (4.12) and (4.13) in the above theorem yields the optimal local convergence estimate

$$
\left\|f-f_{\eta}\right\|_{L^{p}(T)} \leq C_{6}^{\frac{1}{\tau}}\left\|\sqrt{\operatorname{det}\left(d^{2} f\right)}\right\|_{L^{\tau}(T)} N(T, \eta)^{-1} .
$$

In order to obtain the global estimate of Theorem 4.1, we need to be ensured that after sufficiently many steps of the greedy algorithm, the target $f$ can be well approximated by quadratic function $q=q(T)$ on each triangle $T$, so that our local results will apply on such triangles. This is ensured due to the following key result.

Proposition 4.10. Let $f$ be a $C^{2}$ function such that $d^{2} f(x) \geq m I$ for some arbitrary but fixed $m>0$ independent of $x$. Let $\mathcal{T}_{N}$ be the triangulation generated by the greedy algorithm applied to $f$ using the $L^{1}$ decision function given by (2.13). Then

$$
\lim _{N \rightarrow+\infty} \max _{T \in \mathcal{T}_{N}} \operatorname{diam}(T)=0,
$$

i.e., the diameter of all triangles tends to 0 .

Proof. Let $T$ be a triangle with an angle $\theta$ at a vertex $z_{0}$. The other vertices of $T$ can be written as $z_{1}=z_{0}+\alpha u$ and $z_{2}=z_{0}+\beta v$ where $\alpha, \beta \in \mathbb{R}_{+}$and $u, v \in \mathbb{R}^{2}$ are unitary. We assume that $\alpha u$ is the longest edge of $T$, hence $\theta \leq \boldsymbol{\pi} / 2$. Observe that

$$
\rho(T):=\frac{h_{T}^{2}}{|T|}=\frac{\alpha^{2}}{\frac{1}{2} \alpha \beta \sin \theta}=\frac{2 \alpha}{\beta \sin \theta}
$$

and

$$
|u-v|=2 \sin \left(\frac{\theta}{2}\right)=\frac{\sin \theta}{\cos \left(\frac{\theta}{2}\right)}=\frac{2 \alpha}{\beta \rho(T) \cos \left(\frac{\theta}{2}\right)} .
$$

Since $\frac{\sqrt{2}}{2} \leq \cos \left(\frac{\theta}{2}\right)$ we thus obtain

$$
|u-v| \leq \frac{2 \sqrt{2} \alpha}{\beta \rho(T)} \leq \frac{3 \alpha}{\beta \rho(T)} .
$$

We now set $M:=\left\|d^{2} f\right\|_{L^{\infty}(\Omega)}$ and for all $\delta>0$ let

$$
\omega(\delta):=\sup _{z, z^{\prime} \in \Omega,\left\|z-z^{\prime}\right\| \leq \delta}\left\|d^{2} f(z)-d^{2} f\left(z^{\prime}\right)\right\| .
$$

For $t \in \mathbb{R}$, we define

$$
H_{t}^{u}:=d^{2} f_{z_{0}+t u} \text { and } H_{t}^{v}:=d^{2} f_{z_{0}+t v},
$$

and notice that $\left\|H_{t}^{u}-H_{t}^{v}\right\| \leq \omega(t|u-v|)$. Hence, if $0 \leq t \leq \beta$, we have

$$
\left\|H_{t}^{u}-H_{t}^{v}\right\| \leq \omega\left(\frac{3 \alpha}{\rho(T)}\right) .
$$

Furthermore, for all $t$ we have

$$
\begin{aligned}
\left|\left\langle H_{t}^{u} u, u\right\rangle-\left\langle H_{t}^{u} v, v\right\rangle\right| & =\left|\left\langle H_{t}^{u} u, u\right\rangle-\left\langle H_{t}^{u} u-(u-v), u-(u-v)\right\rangle\right| \\
& =\left|2\left\langle H_{t}^{u} u, u-v\right\rangle-\left\langle H_{t}^{u}(u-v), u-v\right\rangle\right| \\
& \leq 2 M|u||u-v|+M|u-v|^{2} \\
& \leq \frac{M}{\beta^{2}}\left(2 \frac{3 \alpha \beta}{\rho(T)}+\left(\frac{3 \alpha}{\rho(T)}\right)^{2}\right) .
\end{aligned}
$$


Applying the identity (2.15) to the edges $e=\alpha u$ and $\beta v$, and using a change of variable, we can write

$$
D_{T}(\alpha u, f)=\int_{\mathbb{R}} \min \{t, \alpha-t\}_{+}\left\langle H_{t}^{u} u, u\right\rangle d t
$$

and

$$
D_{T}(\beta v, f)=\int_{\mathbb{R}} \min \{t, \beta-t\}_{+}\left\langle H_{t}^{v} v, v\right\rangle d t
$$

where we have used the notation $r_{+}:=\max \{r, 0\}$. Hence, noticing that

$$
\int_{\mathbb{R}} \min \{t, \lambda-t\}_{+} d t=\int_{0}^{\lambda} \min \{t, \lambda-t\} d t=\frac{\left(\lambda_{+}\right)^{2}}{4},
$$

and using the previous estimates we obtain

$$
\begin{aligned}
D_{T}(\alpha u, f)-D_{T}(\beta v, f) \\
=\int_{\mathbb{R}}\left(\min \{t, \alpha-t\}_{+}\left\langle H_{t}^{u} u, u\right\rangle-\min \{t, \beta-t\}_{+}\left\langle H_{t}^{v} v, v\right\rangle\right) d t \\
=\int_{\mathbb{R}}\left(\min \{t, \alpha-t\}_{+}-\min \{t, \beta-t\}_{+}\right)\left\langle H_{t}^{u} u, u\right\rangle d t \\
\quad-\int_{\mathbb{R}} \min \{t, \beta-t\}_{+}\left(\left\langle H_{t}^{v} v, v\right\rangle-\left\langle H_{t}^{u} u, u\right\rangle\right) \\
\geq m \int_{\mathbb{R}}\left(\min \{t, \alpha-t\}_{+}-\min \{t, \beta-t\}_{+}\right) d t \\
\quad-\int_{0}^{\beta} \min \{t, \beta-t\}\left(\left|\left\langle H_{t}^{u} u, u\right\rangle-\left\langle H_{t}^{u} v, v\right\rangle\right|+\left|\left\langle\left(H_{t}^{u}-H_{t}^{v}\right) v, v\right\rangle\right|\right) d t \\
\geq m \frac{\alpha^{2}-\beta^{2}}{4}-\frac{M}{4}\left(2 \frac{3 \alpha \beta}{\rho(T)}+\left(\frac{3 \alpha}{\rho(T)}\right)^{2}\right)-\frac{\beta^{2}}{4} \omega\left(\frac{3 \alpha}{\rho(T)}\right) \\
\geq m \frac{\alpha^{2}-\beta^{2}}{4}-\frac{\alpha^{2}}{4}\left(\frac{6}{\rho(T)}+\frac{9}{\rho(T)^{2}}+\omega\left(\frac{3 \alpha}{\rho(T)}\right)\right),
\end{aligned}
$$

where we have used the fact that $\alpha>\beta$ in the last line. We can therefore write

$$
D_{T}(\alpha u, f)-D_{T}(\beta v, f) \geq \frac{m}{4}\left(\alpha^{2}\left(1-\tilde{\omega}\left(\frac{1}{\rho(T)}\right)\right)-\beta^{2}\right),
$$

where we have set

$$
\tilde{\omega}(\delta):=\frac{1}{m}\left(6 \delta+9 \delta^{2}+\omega(3 \operatorname{diam}(\Omega) \delta)\right) .
$$

The inequality (4.15) shows that $d_{T}(\cdot, f)$ prescribes a $\tilde{\omega}\left(\frac{1}{\rho(T)}\right)$-near longest edge bisection in the euclidean metric for any triangle $T$. Indeed, if the smaller edge $\beta v$ was selected, we would necessarily have

$$
|\beta v|^{2}=\beta^{2} \geq\left(1-\tilde{\omega}\left(\frac{1}{\rho(T)}\right)\right) \alpha^{2}=\left(1-\tilde{\omega}\left(\frac{1}{\rho(T)}\right)\right)|\alpha u|^{2} .
$$

Notice that $\tilde{\omega}(\delta) \rightarrow 0$ as $\delta \rightarrow 0$.

Since $f$ is strictly convex, there does not exist any triangle $T \subset \Omega$ such that $e_{T}(f)_{p}=0$. Let us assume for contradiction that the diameter of the triangles generated by the greedy algorithm does not tend to zero. Then there exists a sequence $\left(T_{i}\right)_{i \geq 0}$ of triangles such that $T_{i+1}$ is one of the children of $T_{i}$, and $h_{T_{i}} \rightarrow$ 
$d>0$ as $i \rightarrow \infty$, where $h_{T}$ denotes the diameter of a triangle $T$. Since $\left|T_{i}\right| \rightarrow 0$, this also implies that $\rho\left(T_{i}\right) \rightarrow+\infty$ as $i \rightarrow \infty$. We can therefore choose $i$ large enough such that $h_{T_{i}}^{2}<\frac{4}{3} d^{2}$ and $C_{2} \tilde{\omega}\left(\frac{1}{\rho\left(T_{j}\right)}\right) \leq \frac{1}{2}$ for all $j \geq i$, where $C_{2}$ is the constant in Proposition 4.6. According to this proposition, we have

$$
\sigma\left(T_{i+3}\right) \leq \frac{3}{2} \sigma\left(T_{i}\right)
$$

where $\sigma$ stands for $\sigma_{\mathbf{q}}$ in the euclidean case $\mathbf{q}=x^{2}+y^{2}$. On the other hand, we have for any triangle $T, \frac{h_{T}^{2}}{8|T|} \leq \sigma(T) \leq \frac{h_{T}^{2}}{2|T|}$, from which it follows that

$$
h_{T_{i+3}}^{2} \leq 4 \frac{\left|T_{i+3}\right| \sigma\left(T_{i+3}\right)}{\left|T_{i}\right| \sigma\left(T_{i}\right)} h_{T_{i}}^{2} \leq \frac{3}{4} h_{T_{i}}^{2} .
$$

Therefore, $h_{T_{i+3}}<d$ which is a contradiction. This concludes the proof of Proposition 4.10 .

Proof of Theorem 4.1. Since $f \in C^{2}$, an immediate consequence of Proposition 4.10 is that for all $\mu>0$, there exists

$$
N_{1}:=N_{1}(f, \mu)
$$

such that for all $T \in \mathcal{T}_{N_{1}}$, there exists a quadratic function $q_{T}$ such that

$$
d^{2} q_{T} \leq d^{2} f \leq(1+\mu) d^{2} q_{T}
$$

Therefore, our local results apply on all $T \in \mathcal{T}_{N_{1}}$. Specifically, we choose

$$
N_{1}:=N_{1}\left(f, c_{2}\right),
$$

with $c_{2}$ the constant in Theorem 4.9 . We then take

$$
\eta \leq \eta_{0}:=\min _{T \in \mathcal{T}_{N_{1}}}\left\{e_{T}(f)_{p},\left(\frac{|T|}{\sigma_{\mathbf{q}_{T}}(T)^{\lambda}}\right)^{\frac{1}{\tau}} \sqrt{\operatorname{det} \mathbf{q}}\right\} .
$$

We use the notations

$$
f_{\eta}=f_{N}, \quad \mathcal{T}_{\eta}=\mathcal{T}_{N}, \quad N=N(\eta)=\#\left(\mathcal{T}_{\eta}\right)=\#\left(\mathcal{T}_{N}\right),
$$

for the approximants and triangulation obtained by the greedy algorithm with stopping criterion given by the local error $\eta$. Note that $\mathcal{T}_{\eta}$ is a refinement of $\mathcal{T}_{N_{1}}$, since $\eta \leq \min _{T \in \mathcal{T}_{N_{1}}} e_{T}(f)_{p}$, and therefore $N \geq N_{1}$. We obviously have

$$
\left\|f-f_{N}\right\|_{L^{p}} \leq \eta N^{\frac{1}{p}} .
$$

Using Theorem 4.9, we also have

$$
N=\sum_{T \in \mathcal{T}_{N_{1}}} N(T, \eta) \leq C_{6} \eta^{-\tau}\left\|\sqrt{\operatorname{det}\left(d^{2} f\right)}\right\|_{L^{\tau}(\Omega)}^{\tau},
$$

and therefore

$$
\left\|f-f_{N}\right\| \leq C_{6}^{\frac{1}{\tau}}\left\|\sqrt{\operatorname{det}\left(d^{2} f\right)}\right\|_{L^{\tau}(\Omega)} N^{-1},
$$

which is the claimed estimate. Since we have assumed $\eta \leq \eta_{0}$, this estimate holds for

$$
N>N_{0},
$$

where $N_{0}$ is largest value of $N$ such that $e_{T}(f)_{p} \geq \eta_{0}$ for at least one $T \in \mathcal{T}_{N}$. $\diamond$ 
Remark 4.11. In [8] a modification of the algorithm is proposed so that its convergence in the $L^{p}$ norm is ensured for any function $f \in L^{p}(\Omega)$ (or $f \in C(\Omega)$ when $p=\infty)$. However, this modification is not needed in the proof of Theorem 4.1, due to the assumption that $f$ is convex.

\section{REFERENCES}

[1] V. Babenko, Y. Babenko, A. Ligun and A. Shumeiko, On asymptotical behavior of the optimal linear spline interpolation error of $C^{2}$ functions, East J. Approx. 12(1), 71-101, 2006. MR.2294672 (2007m:41008)

[2] P. Binev, W. Dahmen, R. DeVore and P. Petrushev, Approximation classes for adaptive methods, Serdica Math. J. 28, 391-416, 2002. MR.1965238 (2004b:65176)

[3] H. Borouchaki, P.J. Frey, P.L. George, P. Laug and E. Saltel, Mesh generation and mesh adaptivity: theory, techniques, in Encyclopedia of computational mechanics, E. Stein, R. de Borst and T.J.R. Hughes ed., John Wiley \& Sons Ltd., 2004.

[4] W. Cao, On the error of linear interpolation and the orientation, aspect ratio, and internal angles of a triangle, SIAM J. Numer. Anal. 43(1), 19-40, 2005. MR.2177954 (2006k:65023)

[5] L. Chen, On minimizing the linear interpolation error of convex quadratic functions, East Journal of Approximation 14(3), 271-284, 2008. MR2441084 (2009h:41002)

[6] L. Chen, Mesh smoothing schemes based on optimal Delaunay triangulations, in 13th International Meshing Roundtable, 109-120, Williamsburg, VA, Sandia National Laboratories, 2004.

[7] L. Chen, P. Sun and J. Xu, Optimal anisotropic meshes for minimizing interpolation error in $L^{p}$-norm, Math. of Comp. 76, 179-204, 2007. MR2261017 (2008e:65385)

[8] A. Cohen, N. Dyn, F. Hecht and J.-M. Mirebeau, Adaptive multiresolution analysis based on anisotropic triangulations, Math. of Comp. 81, 789-810, 2012.

[9] R. DeVore, Nonlinear approximation, Acta Numerica 51-150, 1998 MR.1689432 (2001a:41034)

[10] J.-M. Mirebeau, Optimal bidimensional finite element meshes, preprint, Laboratoire J.-L. Lions.

[11] M.C. Rivara, New longest-edge algorithms for the refinement and/or improvement of unstructured triangulations, Int. J. Num. Methods 40, 3313-3324, 1997. MR1471613

Laboratoire Jacques Louis Lions, Université Pierre et Marie Curie, 4, Place Jussieu, 75005 PARIS, FRANCE

E-mail address: mirebeau@ann.jussieu.fr

Laboratoire Jacques Louis Lions, Université Pierre et Marie Curie, 4, Place Jussieu, 75005 PARIS, France-

E-mail address: cohen@ann.jussieu.fr 